\title{
Contact lines over random topographical substrates. Part 2. Dynamics
}

\author{
NIKOS SAVVA ${ }^{1}$, GRIGORIOS A. PAVLIOTIS \\ AND SERAFIM KALLIADASIS ${ }^{1} \dagger$ \\ ${ }^{1}$ Department of Chemical Engineering, Imperial College London, London SW7 2AZ, UK \\ ${ }^{2}$ Department of Mathematics, Imperial College London, London SW7 2AZ, UK
}

(Received 23 March 2010; revised 22 October 2010; accepted 16 November 2010; first published online 11 February 2011)

We examine the dynamics of a two-dimensional droplet spreading over a random topographical substrate. Our analysis is based on the formalism developed in Part 1 of this study, where a random substrate was modelled as band-limited white noise. The system of integrodifferential equations for the motion of the contact points over deterministic substrates derived by Savva and Kalliadasis (Phys. Fluids, vol. 21, 2009, 092102) is applicable to the case of random substrates as well. This system is linearized for small substrate amplitudes to obtain stochastic differential equations for the droplet shift and contact line fluctuations in the limit of shallow and slowly varying topographies. Our theoretical predictions for the time evolution of the statistical properties of these quantities are verified by numerical experiments. Considering the statistics of the dynamics allows us to fully address the influence of the substrate variations on wetting. For example, we demonstrate that the droplet wets the substrate less as the substrate roughness increases, illustrating also the possibility of a substrate-induced hysteresis effect. Finally, the analysis of the long-time limit of spreading dynamics for a substrate represented by a band-limited white noise is extended to arbitrary substrate representations. It is shown that the statistics of spreading is independent of the characteristic length scales that naturally arise from the statistical properties of a substrate representation.

Key words: contact lines, drops, thin films

\section{Introduction}

It is a fundamental problem to understand how deterministic or random heterogeneities influence the characteristics of contact line propagation, e.g. speed and location. Theoretical studies on droplet spreading, a simple prototype for the study of contact line motion, have primarily focused on ideally flat substrates (e.g. Hocking 1983) often in the presence of other effects such as chemical heterogeneities, evaporation and thermocapillarity (e.g. Ehrhard \& Davis 1991; Schwartz 1998; Sodtke, Ajaev \& Stephan 2008; Rednikov, Rossomme \& Colinet 2009).

The effects of substrate topography on droplet spreading dynamics, albeit known from experiments with highly irregular micro-scale features to be significant (Cazabat \& Cohen-Stuart 1986), have received far less attention. The few theoretical studies have focused on deterministic substrates. For example, Gramlich, Mazouchi \& 
Homsy (2004) examined the motion of a two-dimensional contact line over a topographical feature (trench or mound) by solving numerically the full Stokes equations and employing numerical slip at the contact line. Gaskell et al. (2004) examined droplet spreading over a rectangular step by solving numerically the longwave approximation with the constant-thickness precursor film model, often used in spreading studies (see e.g. Troian et al. 1989; Schwartz \& Eley 1998; Kalliadasis 2000). Recently, Savva \& Kalliadasis (2009) examined theoretically two-dimensional droplet (referred to hereafter simply as 'droplet') spreading on spatially varying deterministic topographical substrates. They utilized the long-wave approximation with a slip model and through a singular perturbation method they obtained a system of integrodifferential equations (IDEs) for the evolution of the two moving fronts. The restriction to two dimensions implies that there are no transverse variations, essentially treating the contact lines as a set of two points.

As far as the influence of random substrates on spreading dynamics is concerned, the few previous studies on the subject are mostly based on ad hoc modelling ideas and postulated equations (Moulinet, Guthmann \& Rolley 2002; Tanguy \& Vettorel 2004; Nikolayev 2005; Katzav et al. 2007). To date, no attempt has been made to quantify the statistics of the dynamics on random substrates through a systematic treatment that relies solely on hydrodynamic principles. Considering the statistics of the dynamics also allows us to fully assess the influence of random spatial heterogeneities on wetting.

Our starting point is the set of IDEs developed by Savva \& Kalliadasis (2009). Following the formalism in Savva, Pavliotis \& Kalliadasis (2011, hereafter referred to as Part 1), the topographical substrate is taken to be band-limited white noise. We also assume that characteristic variations of the substrate are much larger than the slip length. The governing equations are given in $\S 2$ while in $\S 3$ they are linearized for small values of the substrate amplitude to obtain a set of evolution equations for the contact line fluctuations and droplet shift. In $\S 4$ we examine the dynamics of the droplet shift and deduce its behaviour analytically through early- and long-time asymptotics. In $\S 5$ we assess the effects of substrate roughness on wetting, by considering the statistics of the contact line fluctuation. In $\S 6$ we examine effects such as droplet equilibria on 'very' rough substrates and a substrate-induced hysteresis effect, demonstrating also the possibility of a stick-slip behaviour that is commonly reported in experiments. Finally, in $\S 7$ we generalize our analysis of spreading on a substrate represented as band-limited white noise to arbitrary substrate representations. A discussion and summary of our results is offered in $\S 8$.

\section{Problem formulation}

We follow Part 1 and represent the substrate as a stationary random function given by a trigonometric series and defined in terms of a characteristic amplitude and wavenumber. Its form is re-written here for the reader's convenience,

$$
\eta(x)=\frac{\eta_{0}}{\sqrt{N}} \sum_{m=1}^{N}\left[\alpha_{m} \sin \frac{k_{0} m x}{N}+\beta_{m} \cos \frac{k_{0} m x}{N}\right],
$$

where $\eta_{0}$ and $k_{0}$ are the characteristic amplitude and wavenumber of the substrate, respectively, and $N$ is a large positive integer. Again, $\alpha_{m}$ and $\beta_{m}$ are statistically independent, normal random variables with $\left\langle\alpha_{m}^{2}\right\rangle=\left\langle\beta_{m}^{2}\right\rangle=1$.

Our starting point is the recent study by Savva \& Kalliadasis (2009) on droplet spreading over deterministic topographical substrates. For the sake of clarity and 
completeness we briefly review their study. These authors utilized a slip model and a long-wave expansion in the Stokes flow regime, i.e. assumed slow flows, small contact angles and strong surface tension effects, obtaining a single equation for the evolution of the droplet thickness $H(x, t)$ over a smooth substrate $\eta(x)$. The spatial coordinate, $x$, and time, $t$, are made dimensionless by the characteristic length scale $L=\sqrt{A /\left(2 \tan \alpha_{s}\right)}$ and time $\tau=3 \mu L /\left(\gamma \tan ^{3} \alpha_{s}\right)$, respectively, where $A$ is the droplet cross-sectional area, $\alpha_{s}$ is the equilibrium contact angle, and $\mu, \gamma$ are is the fluid viscosity and surface tension, respectively. Note that $H(x, t)$ and $\eta(x)$ are scaled by $L \tan \alpha_{s}$. The dimensionless form of the free-surface evolution equation is

$$
H_{t}+\partial_{x}\left[H^{2}(H+\lambda) \partial_{x}^{3}(H+\eta)\right]=0,
$$

where $\lambda \ll 1$ is the non-dimensional slip length, scaled by $L \tan \alpha_{s} / 3$, that originates from the Navier model imposed to make the stress singularity that occurs at the moving contact line integrable (Huh \& Scriven 1971). Unlike the computationally advantageous precursor film model mentioned in $\S 1$, here we maintain a sharp contact line.

Let $a(t)$ and $b(t)$ be the right and left contact points, respectively. Equation (2.2) must be solved subject to the volume constraint

$$
\int_{b}^{a} H(x, t) \mathrm{d} x=2
$$

and the boundary conditions at the two contact points that the droplet thickness vanishes and the angle the free surface makes with the substrate remains equal to its static value, $\alpha_{s}$, so that

$$
\left.\partial_{x} H\right|_{x=a}=-\tan \theta_{a} \quad \text { and }\left.\quad \partial_{x} H\right|_{x=b}=+\tan \theta_{b},
$$

with

$$
\tan \theta_{a}=\frac{1+\eta_{a}^{\prime 2} \tan ^{2} \alpha_{s}}{1+\eta_{a}^{\prime} \tan ^{2} \alpha_{s}} \quad \text { and } \tan \theta_{b}=\frac{1+\eta_{b}^{\prime 2} \tan ^{2} \alpha_{s}}{1-\eta_{b}^{\prime} \tan ^{2} \alpha_{s}}
$$

where we use $\left.\partial_{x} \eta\right|_{x=c}=\eta_{c}^{\prime}$. To be consistent with the long-wave expansion, we should have taken $\tan \theta_{a}=\tan \theta_{b}=1$, given that $\tan \alpha_{s} \ll 1$ by assumption. However, we retain the full expression to exactly force the contact angle conditions. In principle, we rarely see a difference between simulations that use the two different forms, but the droplet may be driven to a different equilibrium if initially it is close to a boundary that separates the basins of attraction of two stable equilibria.

For quasi-static spreading, asymptotic matching of the solution in the bulk of the fluid with the solutions in the vicinity of the contact lines yields the following IDEs for the time evolution of the two moving fronts:

$$
\dot{a}=+\frac{\delta_{a} M_{b}+\delta_{b} \phi_{a} M_{0}}{M_{a} M_{b}-\phi_{a} \phi_{b} M_{0}^{2}} \quad \text { and } \quad \dot{b}=-\frac{\delta_{b} M_{a}+\delta_{a} \phi_{b} M_{0}}{M_{a} M_{b}-\phi_{a} \phi_{b} M_{0}^{2}},
$$

where the dots denote time differentiation. Here we identify

$$
\begin{aligned}
& \delta_{a}=\frac{1}{3}\left(\phi_{a}^{3}-\theta_{a}^{3}\right), \\
& \delta_{b}=\frac{1}{3}\left(\phi_{b}^{3}-\theta_{b}^{3}\right),
\end{aligned}
$$




$$
\begin{aligned}
& M_{a}=\ln \left(\theta_{a} \frac{a-b}{\lambda}\right)+\int_{-1}^{+1} \frac{1}{1-y}\left[\frac{\phi_{a}^{3}(a-b)^{3}\left(1-y^{2}\right)^{4}}{128 H_{0}^{3}(1-y)}-1\right] \mathrm{d} y, \\
& M_{b}=\ln \left(\theta_{b} \frac{a-b}{\lambda}\right)+\int_{-1}^{+1} \frac{1}{1+y}\left[\frac{\phi_{b}^{3}(a-b)^{3}\left(1-y^{2}\right)^{4}}{128 H_{0}^{3}(1+y)}-1\right] \mathrm{d} y, \\
& M_{0}=\frac{\phi_{a} \phi_{b}(a-b)^{3}}{128} \int_{-1}^{+1} \frac{\left(1-y^{2}\right)^{3}}{H_{0}^{3}} \mathrm{~d} y,
\end{aligned}
$$

where

$$
\begin{aligned}
\phi_{a} & =\frac{2}{a-b}\left[\frac{6}{a-b}+3 \bar{\eta}-2 \eta_{a}-\eta_{b}+\frac{1}{2} \eta_{a}^{\prime}(a-b)\right], \\
\phi_{b} & =\frac{2}{a-b}\left[\frac{6}{a-b}+3 \bar{\eta}-2 \eta_{b}-\eta_{a}-\frac{1}{2} \eta_{b}^{\prime}(a-b)\right],
\end{aligned}
$$

correspond to the 'mesoscopic' contact angles at $x=a$ and $b$, respectively, observed at distances at which the substrate features are sufficiently resolved (a schematic illustrating the different contact lines is given in the study of Savva \& Kalliadasis 2009) and $y$, a coordinate introduced to facilitate the analysis, is defined from $x=(a-b) y / 2+(a+b) / 2$ (so that the domain $a \leqslant x \leqslant b$ is mapped to $-1 \leqslant y \leqslant+1$ ). Also,

$$
H_{0}(y)=\frac{3}{2}\left[\frac{2}{a-b}+\bar{\eta}-\frac{1}{2}\left(\eta_{a}+\eta_{b}\right)\right]\left(1-y^{2}\right)+\frac{1}{2}\left[\eta_{a}(1+y)+\eta_{b}(1-y)\right]-\eta(x)
$$

is the leading-order solution in the bulk of the droplet with $\eta_{a}=\eta(a), \eta_{b}=\eta(b)$ and $\bar{\eta}=\int_{b}^{a} \eta(\xi) \mathrm{d} \xi /(a-b)$. Savva \& Kalliadasis (2009) confirmed the validity of $(2.6 a, b)$ through detailed comparisons with numerical solutions to the full equation (2.2). In addition, they examined many qualitative features of droplet spreading, but they restricted their attention to deterministic substrates only.

\section{Derivation of leading-order equations for droplet shift and contact line fluctuations}

Analytical progress can be made by assuming a small substrate amplitude, which then allows us to linearize $(2.6 a, b)$ around the flat-substrate case. We note that even if we do so, because of the spatial dependence of the noise, the contact line locations enter $(2.6 a, b)$ in a highly nonlinear fashion, thus precluding the use of the standard Langevin-Fokker-Planck formalism often employed to study randomly perturbed dynamical systems (e.g. Gardiner 1985). Proceeding as in Part 1, we introduce the droplet shift, $\ell$, and contact line fluctuation, $\varepsilon$, which allows us to split the droplet motion into two separate components. The former accounts for a sliding motion along the topographical features whereas the latter accounts for deviations from the droplet radius of the flat-substrate spreading dynamics. This distinction results into the following dynamical system:

$$
\dot{\varepsilon}=\frac{1}{2}(\dot{a}-\dot{b})-\dot{x}_{0}, \quad \dot{\ell}=\frac{1}{2}(\dot{a}+\dot{b}),
$$

where $x_{0}(t)$ is the flat-substrate droplet radius given by the solution to the differential equation

$$
3 \dot{x}_{0} \ln \frac{2 x_{0}}{\lambda e^{2}}=\frac{27}{x_{0}^{6}}-1,
$$


which approaches $\sqrt{3}$ in the long-time limit. In order to express the right-hand sides of $(3.1 a, b)$ in terms of $\ell$ and $\varepsilon$, we employ a linearization procedure which takes $\tan \theta_{a}=\tan \theta_{b}=1$ and treats $\varepsilon$ as $O\left(\eta_{0}\right)$, retaining only the linear terms in $\eta_{0}$ and $\varepsilon$. As with the statics, the linearization is valid provided that

$$
\eta_{0} k_{0}^{2} \ll 1 .
$$

The derivation of the linearized equations is rather lengthy and tedious, and for the sake of brevity of presentation we omit its details here. However, through certain algebraic manipulations more compact and manageable expressions can be obtained, as for example the symmetrization of the integrand of $M_{0}$. By neglecting the higherorder terms in $\eta_{0}$, we obtain at the end a stochastic differential equation for $\varepsilon$ of the form

$$
\dot{\varepsilon}+A(t) \varepsilon=\frac{\eta_{0}}{\sqrt{N}} \sum_{m=1}^{N}\left(\alpha_{m} \sin k_{m} \ell+\beta_{m} \cos k_{m} \ell\right) B\left(t, k_{m} x_{0}\right),
$$

where

$$
\begin{gathered}
A(t)=\frac{1}{x_{0} \ln \frac{2 x_{0}}{\lambda e^{2}}}\left(\dot{x}_{0}+\frac{54}{x_{0}^{6}}\right) \\
B(t, \xi(t))=\frac{x_{0}}{\ln \frac{2 x_{0}}{\lambda e^{2}}}\left\{\frac{27}{x_{0}^{6}}\left(\operatorname{sinc} \xi-\cos \xi-\frac{1}{3} \xi \sin \xi\right)\right. \\
\left.+\dot{x}_{0}[(\xi \sin \xi-\cos \xi) \operatorname{Cin} 2 \xi+(\xi \cos \xi+\sin \xi) \operatorname{Si} 2 \xi-3 \xi \sin \xi]\right\} .
\end{gathered}
$$

Here we set

$$
\operatorname{sinc} x=\frac{\sin x}{x}, \quad \text { Si } x=\int_{0}^{x} \operatorname{sinc} x^{\prime} \mathrm{d} x^{\prime}, \quad \operatorname{Cin} x=\int_{0}^{x} \frac{1-\cos x^{\prime}}{x^{\prime}} \mathrm{d} x^{\prime},
$$

corresponding to the cardinal sine function, sine and cosine integrals, respectively (Abramowitz \& Stegun 1964). Since (3.4) is linear in $\varepsilon$, we can obtain the following explicit solution:

$$
\varepsilon(t)=\frac{\eta_{0}}{\sqrt{N}} \sum_{m=1}^{N} \int_{0}^{t}\left(\alpha_{m} \sin k_{m} \ell\left(t^{\prime}\right)+\beta_{m} \cos k_{m} \ell\left(t^{\prime}\right)\right) B\left(t^{\prime}, k_{m} x_{0}\right) \exp \left(-\int_{t^{\prime}}^{t} A\left(t^{\prime \prime}\right) \mathrm{d} t^{\prime \prime}\right) \mathrm{d} t^{\prime} .
$$

For the droplet shift we obtain the stochastic differential equation

$$
\dot{\ell}=\frac{\eta_{0}}{\sqrt{N}} \sum_{m=1}^{N}\left(\alpha_{m} \cos k_{m} \ell-\beta_{m} \sin k_{m} \ell\right) C\left(t, k_{m} x_{0}\right),
$$

where, again, the higher-order terms are neglected and

$$
\begin{aligned}
C(t, \xi(t))=\frac{x_{0}}{\ln \frac{2 x_{0}}{\lambda}} & \left\{\frac{9}{x_{0}^{6}}(\xi \cos \xi-\sin \xi)\right. \\
& \left.+\dot{x}_{0}\left[\xi\left(\frac{7}{3}-\operatorname{Cin} 2 \xi\right) \cos \xi+\left(\xi \operatorname{Si} 2 \xi-\frac{7}{3}\right) \sin \xi\right]\right\} .
\end{aligned}
$$


It is clear that in the above system of differential equations, (3.4) and (3.9), the time dependence enters $A(t, \xi(t)), B(t, \xi(t))$ and $C(t, \xi(t))$ through $x_{0}(t)$ and its time derivative.

Evidently, the solution to (3.9) cannot be given in explicit form. However, since at $t=0$ we have that $\ell=0$, we may obtain its early-time behaviour by expanding the trigonometric functions for small $\ell$, which leads to

$$
\dot{\ell}=\frac{\eta_{0}}{\sqrt{N}} \sum_{m=1}^{N}\left(\alpha_{m}-\beta_{m} k_{m} \ell\right) C\left(t, k_{m} x_{0}\right)+O\left(\ell^{2}\right) .
$$

By neglecting the higher-order terms, we get a linear differential equation for $\ell$ that may be solved explicitly to yield

$$
\ell(t)=\frac{\eta_{0}}{\sqrt{N}} \sum_{m=1}^{N} \int_{0}^{t} \alpha_{m} C\left(t^{\prime}, k_{m} x_{0}\right) \exp \left(-\frac{\eta_{0}}{\sqrt{N}} \sum_{n=1}^{N} \int_{t^{\prime}}^{t} \beta_{n} k_{n} C\left(t^{\prime \prime}, k_{n} x_{0}\right) \mathrm{d} t^{\prime \prime}\right) \mathrm{d} t^{\prime}
$$

Similarly, (3.8) becomes

$$
\varepsilon(t)=\frac{\eta_{0}}{\sqrt{N}} \sum_{m=1}^{N} \int_{0}^{t}\left(\alpha_{m} k_{m} \ell\left(t^{\prime}\right)+\beta_{m}\right) B\left(t^{\prime}, k_{m} x_{0}\right) \exp \left(-\int_{t^{\prime}}^{t} A\left(t^{\prime \prime}\right) \mathrm{d} t^{\prime \prime}\right) \mathrm{d} t^{\prime} .
$$

The analysis that follows is based on the system of differential equations (3.2), (3.4) and (3.9) and aims to characterize the statistics of $\varepsilon$ and $\ell$. Our theoretical predictions will be compared with numerical solutions to the IDEs in $(2.6 a, b)$ by considering typically $2 \times 10^{4}$ realizations from each substrate family. At $t=0$, we take $x_{0}(0)=1$, $\varepsilon(0)=0$ and $\ell(0)=0$. Given also the generally weak dependence of the dynamics on the slip length (Hocking 1983), in all computations we fix $\lambda=10^{-4}$ as well as the static contact angle $\alpha_{s}=10^{\circ}$ in order to focus only on the effects of substrate topography. The value of the static contact angle in particular, is not expected to play an important role. It must be small to conform with the long-wave expansion, and besides it enters the boundary conditions $(2.4 a, b)$ as $\tan ^{2} \alpha_{s}$.

Typical evolution curves for $\varepsilon(t)$ and $\ell(t)$ are shown in figure 1 for three random substrate realizations with $\eta_{0}=5 \times 10^{-4}$ and $k_{0}=15$. The plots in figure $1(a-c)$ depict $\varepsilon$ as a function of $t$ for each of the substrate realizations and the plots in figure $1(d-f)$ show the corresponding evolution curves for $\ell$. The solid lines are the solutions to the system of IDEs, $(3.1 a, b)$, the dashed curves are the solutions to the linearized equations, (3.8) and (3.9), whereas the dotted lines correspond to the early-time asymptotic curves, (3.12) and (3.13). Provided that (3.3) is valid, our linearized theory typically yields excellent results, especially at the onset of spreading. In the majority of our random simulations, we observe behaviours similar to figures $1(a)$ and $1(d)$, where we notice an overall good agreement for all times for the linearized equations, but the early-time asymptotics fail to capture the long-time behaviour. It is also quite common to find substrate realizations for which the agreement is excellent for all times, even for the early-time asymptotics, as shown in figures $1(b)$ and $1(e)$, where all three curves are nearly indistinguishable. However, in some rare cases illustrated by figures $1(c)$ and $1(f)$, our theory fails to predict the behaviour at long times, and there is a clear deviation towards different equilibria. This is possibly due to the nonlinear interplay of our small parameter, $\eta_{0} k_{0}^{2}$, with $\dot{x}_{0}$ when the latter is also small. However, since such cases are not as common as the other two cases, we do not believe that they can affect the overall statistics and for this reason we did not deem it necessary to exclude such situations. Having verified the generally excellent 

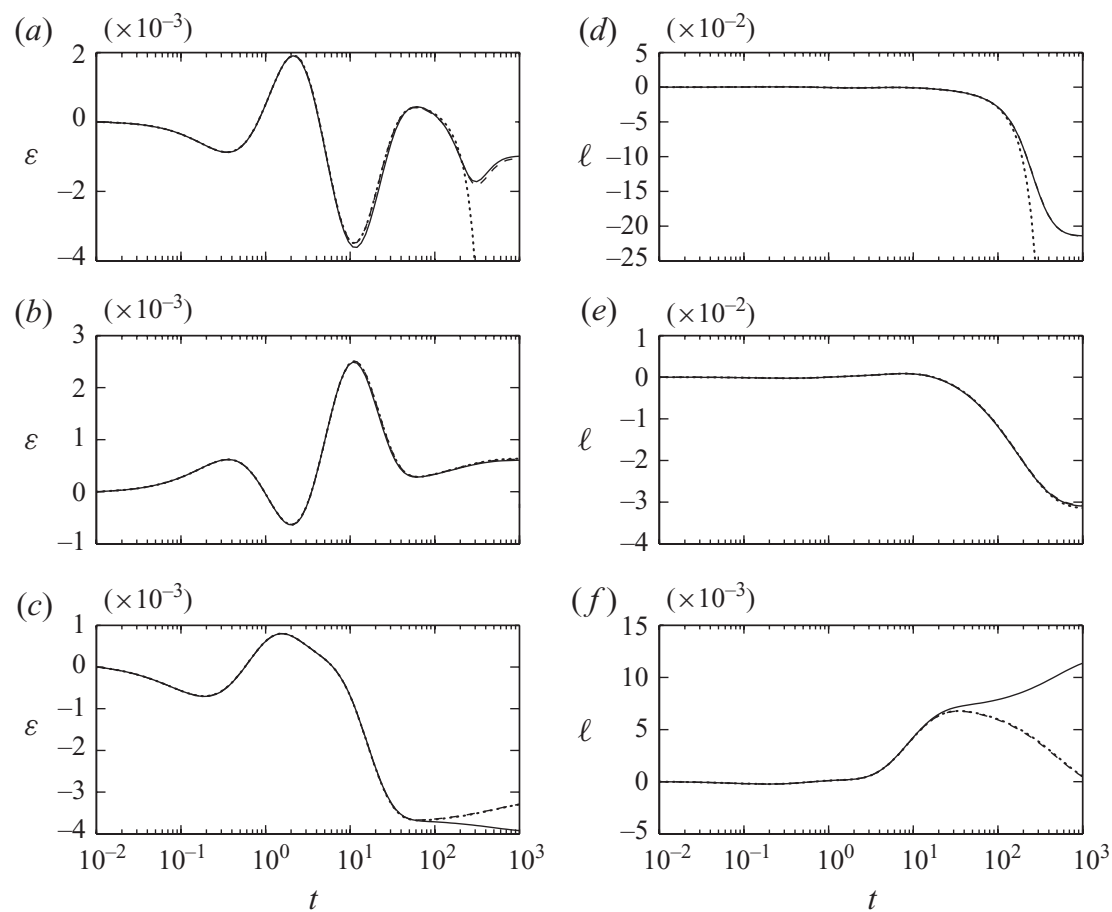

FIGURE 1. Time evolution of $\varepsilon$ and $\ell$ for $\eta_{0}=5 \times 10^{-4}$ and $k_{0}=15$ for three substrate realizations comparing the solutions to the IDEs (solid lines), $(3.1 a, b)$, the solutions to the linearized equations (dashed lines), (3.4) and (3.9), and the early-time approximation (dotted lines), (3.12) and (3.13). The plots in $(a-c)$ show $\varepsilon(t)$ for each of the three random substrate realizations and those in $(d-f)$ show the corresponding curves for $\ell(t)$.

performance of our linear theory, we proceed to examine the statistics of $\ell$, since its leading-order dynamics does not depend on $\varepsilon$, as suggested by (3.9).

\section{Droplet shift dynamics}

As mentioned above, (3.9) cannot be solved analytically and a theoretical assessment of the statistics of $\ell$ for all times is not possible. As a matter of fact, we are bound to encounter similar difficulties in assessing the statistical properties of $\ell$ as we did for its static equilibria in Part 1. Nevertheless, it is still possible to examine analytically the early-/long-time asymptotics of (3.9).

Owing to symmetry, we expect that $\langle\ell(t)\rangle=0 \forall t$, since the droplet should not preferentially slide on either side of the topography with respect to our chosen origin. Clearly, the effects of the substrate characteristics on wetting do not depend explicitly on the statistics of $\ell$, since only the statistics of the droplet radius are needed to calculate, for example, an apparent contact angle. However, the droplet shift is expected to influence wetting indirectly as the dependence of $\varepsilon$ on $\ell$ in (3.4) suggests.

While considering the statics in Part 1, we concluded that the statistics of $\ell$ do not depend on $\eta_{0}$. We note from (3.9), however, that the speed at which the droplet slides is influenced by $\eta_{0}$. In fact, due to the linearity of (3.9) on $\eta_{0}$, the equilibrium tends to be attained at a faster rate when the topography amplitude increases.

Hence, at the onset we see from (3.12) that $\ell$ is given as an infinite sum of random variables with well-prescribed moments and by the central limit theorem (Breiman 
1992), it is therefore well approximated as a normal variable. At later times, for which $\dot{\ell} \rightarrow 0$, the early-time asymptotics are expected to fail given that (3.11) predicts that $\ell$ should approach a ratio of two normal variables, i.e. a Cauchy random variable, whose mean and variance are not defined. We have seen in Part 1 , however, that the equilibria of $\ell$ have a well-defined variance, albeit with a probability density that cannot be determined analytically. Hence, we can assess the region of validity of (3.12) by computing the variance of $\ell$ and compare it with results from numerical experiments. Consider

$$
\begin{aligned}
\ell^{2} & =\frac{\eta_{0}^{2}}{N} \sum_{q=1}^{N} \sum_{m=1}^{N} \alpha_{q} \alpha_{m} \int_{0}^{t} \int_{0}^{t} C\left(t^{\prime}, k_{m} x_{0}\right) C\left(t^{\prime \prime}, k_{n} x_{0}\right) \\
& \times \exp \left(-\frac{\eta_{0}}{\sqrt{N}} \sum_{n=1}^{N} \beta_{n}\left[\int_{t^{\prime}}^{t} k_{q} C\left(t^{\prime \prime \prime}, k_{n} x_{0}\right) \mathrm{d} t^{\prime \prime \prime}+\int_{t^{\prime \prime}}^{t} k_{n} C\left(t^{\prime \prime \prime}, k_{n} x_{0}\right) \mathrm{d} t^{\prime \prime \prime}\right]\right) \mathrm{d} t^{\prime} \mathrm{d} t^{\prime \prime} .
\end{aligned}
$$

By taking the ensemble average, we can suppress the summation with respect to $q$ due to the mutual independence of the $\alpha_{m}$ values so that

$$
\begin{aligned}
\operatorname{Var}[\ell] & =\left\langle\ell^{2}\right\rangle=\frac{\eta_{0}^{2}}{N} \sum_{m=1}^{N} \int_{0}^{t} \int_{0}^{t} C\left(t^{\prime}, k_{m} x_{0}\right) C\left(t^{\prime \prime}, k_{q} x_{0}\right) \\
& \times \exp \left(\frac{\eta_{0}^{2}}{2 N} \sum_{n=1}^{N}\left[\int_{t^{\prime}}^{t} k_{n} C\left(t^{\prime \prime \prime}, k_{n} x_{0}\right) \mathrm{d} t^{\prime \prime \prime}+\int_{t^{\prime \prime}}^{t} k_{n} C\left(t^{\prime \prime \prime}, k_{n} x_{0}\right) \mathrm{d} t^{\prime \prime \prime}\right]^{2}\right) \mathrm{d} t^{\prime} \mathrm{d} t^{\prime \prime},
\end{aligned}
$$

where the mean of the exponential term is found using $\left\langle\mathrm{e}^{X}\right\rangle=\mathrm{e}^{\sigma_{X}^{2} / 2}$, for $X$ being a zero-mean normal variable with variance $\sigma_{X}^{2}$. Conversion of the Riemann sums into integrals gives the variance of $\ell$ at early times

$$
\begin{aligned}
\operatorname{Var}[\ell] & =\eta_{0}^{2} \int_{0}^{1} \int_{0}^{t} \int_{0}^{t} C\left(t^{\prime}, k_{0} x_{0} y\right) C\left(t^{\prime \prime}, k_{0} x_{0} y\right) \\
& \times \exp \left(\frac{\eta_{0}^{2} k_{0}^{2}}{2} \int_{0}^{1}\left[D\left(t^{\prime}, q ; t\right)+D\left(t^{\prime \prime}, q ; t\right)\right]^{2} \mathrm{~d} q\right) \mathrm{d} t^{\prime} \mathrm{d} t^{\prime \prime},
\end{aligned}
$$

where the functional $D$ is given by

$$
D(\tau, q ; t)=\int_{\tau}^{t} q C\left(t^{\prime}, q k_{0} x_{0}\left(t^{\prime}\right)\right) \mathrm{d} t^{\prime} .
$$

Figure 2 depicts the evolution of $\sigma_{\ell}=\sqrt{\operatorname{Var}[\ell]}$ as computed from numerical experiments, together with the early-time behaviour predicted by (4.3) for $\eta_{0}=5 \times 10^{-4}$ and $k_{0}=10,20,30$ and 40 . We note that depending on the value of $k_{0}$, the agreement between the linear theory and the numerics can be excellent even up to $t \sim O\left(10^{2}\right)$; after that time the theoretically predicted variance goes to infinity in accord with our earlier discussion that (3.11) predicts a Cauchy variable in the long-time limit. For intermediate times, it is impossible to theoretically predict any of the statistical properties of $\ell$ and we can only resort to a numerical study. Naturally, the tendency of the droplet to slide along the topographical features is reduced as $k_{0}$ increases. This behaviour may be inferred from figure 2, where we see that at the end of computation, at $t=380, \sigma_{\ell}$ is closer to saturating for $k_{0}=40$ compared with $k_{0}=10$ which is still growing. 


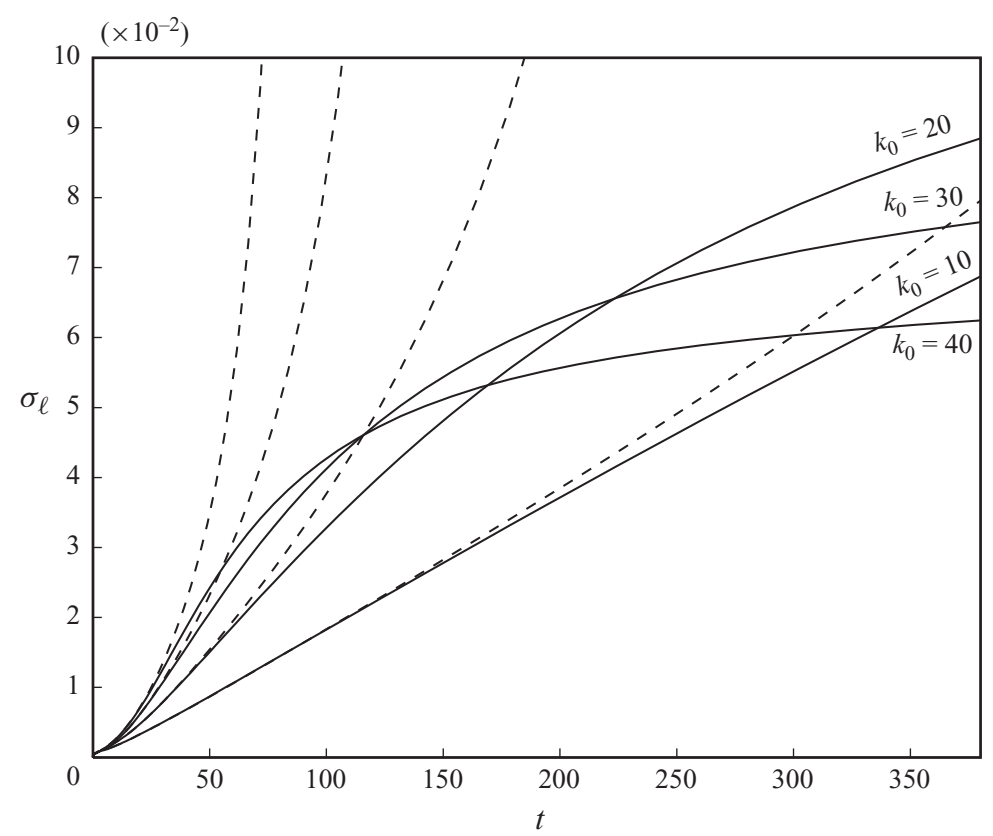

FIgURE 2. Time evolution of $\sigma_{\ell}$ for $\eta_{0}=5 \times 10^{-4}$ and $k_{0}=10,20,30$ and 40 . The solid lines correspond to numerical experiments, whereas the dashed lines to the early-time asymptotics predicted by (4.3).

In the long-time limit, where $\dot{\ell} \rightarrow 0$ and $\ell \rightarrow \ell_{\infty}$, we obtain the following equation for the droplet shift at equilibrium:

$$
\sum_{m=1}^{N}\left(\alpha_{m} \cos k_{m} \ell_{\infty}-\beta_{m} \sin k_{m} \ell_{\infty}\right) G\left(k_{m} \sqrt{3}\right)=0,
$$

which is identical to (3.13) obtained in Part 1 for the droplet statics (as in Part 1, we set $G(\xi)=\xi \cos \xi-\sin \xi$ ). As we have also pointed out in Part 1, (4.5) admits infinitely many solutions as $N \rightarrow \infty$. But now we are dealing with a dynamical system and where contrary to the statics we have an evolution from $\ell=0$ to $\ell_{\infty}$. Hence, we are interested not only in a solution to (4.5) that is closest to $\ell=0$ but also in a solution that corresponds to a stable equilibrium. Again, this is reminiscent of a first-passage problem, but we have the additional constraint that the equilibrium $\ell_{\infty}$ must also be stable. To find conditions for the stability of this equilibrium, we introduce in (3.9) $\ell=\ell_{\infty}+\tilde{\ell}$ where $\tilde{\ell}$ is a small perturbation. Doing so yields the linearized system

$$
\dot{\tilde{\ell}}=-\frac{\eta_{0}}{\sqrt{3} \ln \frac{2 \sqrt{3}}{\lambda}}\left[\sum_{m=1}^{N} k_{m}\left(\alpha_{m} \sin k_{m} \ell_{\infty}+\beta_{m} \cos k_{m} \ell_{\infty}\right) G\left(k_{m} \sqrt{3}\right)\right] \tilde{\ell} .
$$

Linear stability of the equilibrium $\ell=\ell_{\infty}$ requires that

$$
\sum_{m=1}^{N} k_{m}\left(\alpha_{m} \sin k_{m} \ell_{\infty}+\beta_{m} \cos k_{m} \ell_{\infty}\right) G\left(k_{m} \sqrt{3}\right)>0
$$


(a)

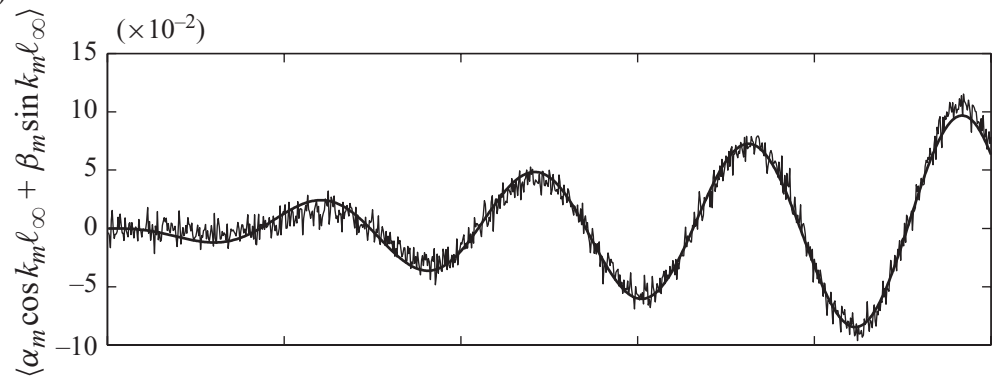

(b)

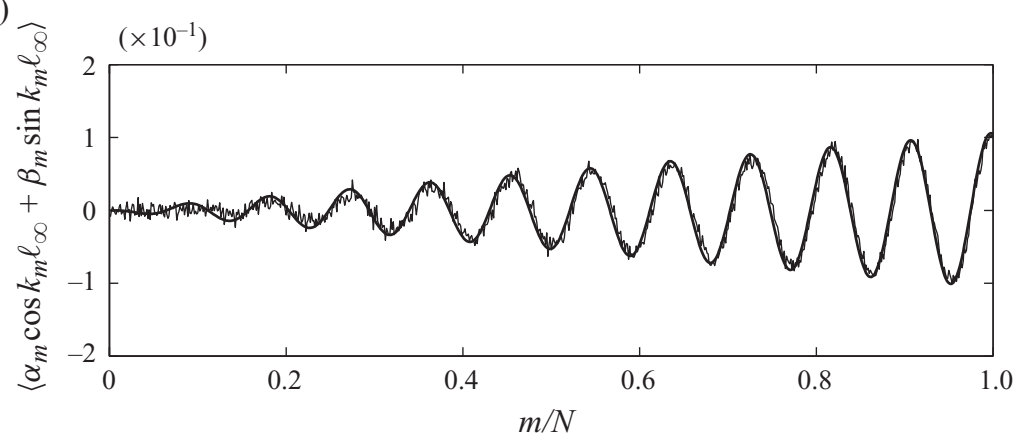

Figure 3. Comparison of $\left\langle\alpha_{m} \sin k_{m} \ell_{\infty}+\beta_{m} \cos k_{m} \ell_{\infty}\right\rangle$ obtained from numerical experiments (thin lines) and the semi-analytical result (4.9) (thick lines) for substrate families with $(a)$ $\eta_{0}=10^{-3}, k_{0}=15, N=900$ and $(b) \eta_{0}=10^{-3}, k_{0}=40$ and $N=800$.

which should also hold for the ensemble average. Hence, we anticipate that the validity of an ensemble average of the above inequality is guaranteed if we have

$$
\left\langle\alpha_{m} \sin k_{m} \ell_{\infty}+\beta_{m} \cos k_{m} \ell_{\infty}\right\rangle \propto \frac{G\left(k_{m} \sqrt{3}\right)}{\sqrt{N}},
$$

so that the summands in (4.7) are all positive. A rigorous theory to support the above claim is presently lacking but our numerical experiments suggest that, provided (3.3) holds, we have

$$
\left\langle\alpha_{m} \sin k_{m} \ell_{\infty}+\beta_{m} \cos k_{m} \ell_{\infty}\right\rangle \approx \sqrt{\frac{3}{N}} \frac{G\left(k_{m} \sqrt{3}\right)}{k_{0}} .
$$

The inverse square-root dependence on the number of harmonics comes as no surprise since as $N$ increases, the final value of $\ell_{\infty}$ becomes 'less dependent' on the individual $\alpha_{m}$ and $\beta_{m}$. At this stage, it is crucial not to take $N \rightarrow \infty$, in which case the right-hand side of (4.9) vanishes, because this relation has important implications in characterizing wetting on random substrates, as it is responsible for a non-zero $\langle\varepsilon\rangle$. To demonstrate the validity of this semi-analytical result, we depict in figure 3 plots of the mean of $\alpha_{m} \sin k_{m} \ell_{\infty}+\beta_{m} \cos k_{m} \ell_{\infty}$ as a function of $m / N$. In figure $3(a)$, $N=900$ harmonics were used with $\eta_{0}=10^{-3}$ and $k_{0}=15$ and in figure $3(b), N=800$ with $\eta_{0}=10^{-3}$ and $k_{0}=40$. Evidently, our numerical experiments (thin lines) closely follow (4.9) (thick lines), even for $k_{0}=40$, where, strictly speaking, (3.3) does not hold. The fluctuations, which appear to persist regardless of the size of $N$, may be possibly attributed to unavoidable roundoff errors in our computations. 


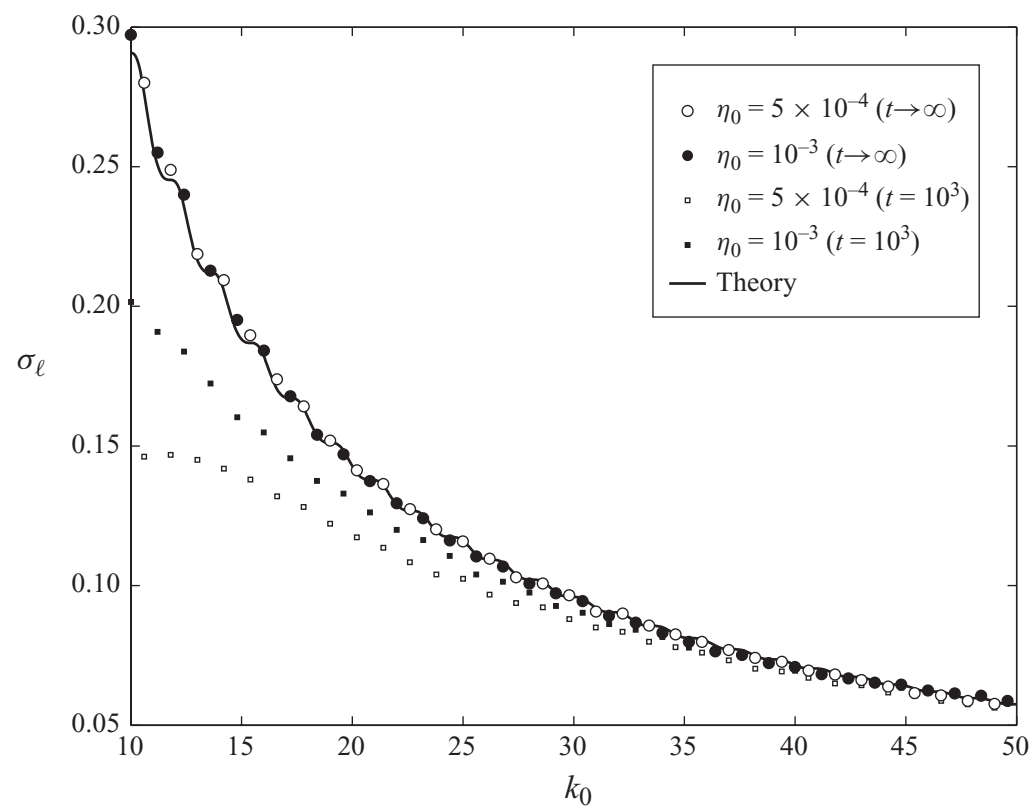

FIgURE 4. Variation of $\sigma_{\ell}$ as a function of $k_{0}$ at equilibrium (circles) and at time $t=1000$ (squares) together with the equilibrium predicted by (4.10). The open and filled symbols correspond to $\eta_{0}=5 \times 10^{-4}$ and $\eta_{0}=10^{-3}$, respectively. In the long-time limit $\sigma_{\ell}$ becomes independent of $\eta_{0}$.

The probability density function of $\ell_{\infty}$ cannot be predicted analytically, but can be nevertheless approximated via Padé approximants as discussed in Part 1. The computation of the variance of $\ell_{\infty}$ is performed in a manner similar to that in Part 1 . We expect that the variance scales with $\langle\Delta \ell\rangle^{2}$, where $\langle\Delta \ell\rangle$ is the mean distance between zeros, given by (4.7) of Part 1 . Since on average, a stable equilibrium is expected to be found within a distance $\langle\Delta \ell\rangle$ from the origin, we can also argue that

$$
\sigma_{\ell_{\infty}}^{2}=\operatorname{Var}\left[\ell_{\infty}\right]=\frac{1}{2}\langle\Delta \ell\rangle^{2} \sim \frac{5 \pi^{2}}{6 k_{0}^{2}}\left[1-2 \operatorname{sinc}\left(2 k_{0} \sqrt{3}\right)\right]+O\left(k_{0}^{-4}\right),
$$

in the limit $k_{0} \gg 1$. It is important to note that $\sigma_{\ell_{\infty}}$ is two times larger than that obtained from statics considerations. This is attributed to the fact that, on average half of the equilibria considered are unstable, whereas in a dynamic setting we are seeking the stable equilibria only. The overall qualitative behaviour is that the droplet slides less as the length scale of substrate variations decreases. Such behaviour is confirmed in figure 4 , where we show the theoretically predicted $\sigma_{\ell_{\infty}}$ as a function of $k_{0}$ together with numerical experiments for substrate families with $\eta_{0}=10^{-3}$ (filled circles) and $\eta_{0}=5 \times 10^{-4}$ (open circles). In figure 4, we also depict by filled and open squares the corresponding $\sigma_{\ell}$ at time $t=1000$. For smaller $\eta_{0}$, the approach to equilibrium is slower compared with that for larger values of $\eta_{0}$, but as $t \rightarrow \infty, \sigma_{\ell}$ eventually becomes independent of $\eta_{0}$. Finally, it is worth noting that our numerical experiments also show that the veracity of (4.10) may extend beyond its regime of validity imposed by (3.3). 


\section{Contact line fluctuation dynamics}

The dynamics of contact line fluctuations can be used to assess the effect of the substrate features on wetting. This can be quantified through the apparent contact angle, $\theta_{\text {app }}$, given by

$$
\theta_{\text {app }}=\frac{3}{\left(x_{0}+\varepsilon\right)^{2}}=\theta_{\text {flat }}\left(1-2 \frac{\varepsilon}{x_{0}}\right)+O\left(\varepsilon^{2} / x_{0}^{2}\right),
$$

where $\theta_{\text {flat }}=3 / x_{0}^{2}$ corresponds to the apparent contact angle for a droplet on an ideally flat substrate. As we have already pointed out in $\S 4$, the dynamics of $\ell$ is required in the computations for $\varepsilon$, because it appears in its evolution equation (3.8).

The central limit theorem is expected to apply in (3.8) and through a similar reasoning as the one offered in $\S 4$ for (3.12), we may conclude that $\varepsilon(t)$ is adequately approximated by a normal variable for all $t$ with a probability density function

$$
p_{\varepsilon}(\epsilon)=\frac{1}{\sqrt{2 \pi \sigma_{\varepsilon}^{2}}} \exp \left(-\frac{\left(\epsilon-\mu_{\varepsilon}\right)^{2}}{2 \sigma_{\varepsilon}^{2}}\right),
$$

where $\mu_{\varepsilon}$ corresponds to its mean and $\sigma_{\varepsilon}^{2}$ to its variance. Hence the statistics of $\varepsilon$ are completely determined by $\mu_{\varepsilon}$ and $\sigma_{\varepsilon}^{2}$. However, the dependence of $\varepsilon$ on $\ell$ complicates matters considerably.

\subsection{Mean of contact line fluctuations}

The present theory cannot predict the time evolution of $\mu_{\varepsilon}$, because to do so we need to determine $\left\langle\alpha_{m} \sin k_{m} \ell+\beta_{m} \cos k_{m} \ell\right\rangle$ as a function of $t$, a task that cannot be carried out analytically. However, we can deduce a differential equation for the early-time behaviour of $\mu_{\varepsilon}$, using the early-time behaviour of $\ell(t)$, prescribed by (3.12). Since at the onset $\ell$ is small, we expand (3.4) for small $\ell$ and use (3.12) to finally obtain

$$
\begin{aligned}
& \dot{\mu}_{\varepsilon}+A(t) \mu_{\varepsilon}=\eta_{0}^{2} k_{0} \int_{0}^{1} y B\left(t, k_{0} x_{0} y\right) \\
& \quad \times \int_{0}^{t} C\left(t^{\prime}, k_{0} x_{0} y\right) \exp \left(\frac{\eta_{0}^{2} k_{0}^{2}}{2} \int_{0}^{1}\left[q \int_{t^{\prime}}^{t} C\left(\tau, k_{0} x_{0} q\right) \mathrm{d} \tau\right]^{2} \mathrm{~d} q\right) \mathrm{d} t^{\prime} \mathrm{d} y,
\end{aligned}
$$

upon taking an ensemble average of all substrate realizations. Again, this is a linear equation whose solution may be written explicitly as

$$
\begin{aligned}
\mu_{\varepsilon}(t)= & \eta_{0}^{2} k_{0} \int_{0}^{t} \exp \left[-\int_{t^{\prime}}^{t} A(s) \mathrm{d} s\right] \int_{0}^{t^{\prime}} \exp \left(\frac{\eta_{0}^{2} k_{0}^{2}}{2} \int_{0}^{1}\left[q \int_{t^{\prime \prime}}^{t^{\prime}} C\left(\tau, k_{0} x_{0} q\right) \mathrm{d} \tau\right]^{2} \mathrm{~d} q\right) \\
& \times \int_{0}^{1} y B\left(t^{\prime}, k_{0} x_{0} y\right) C\left(t^{\prime \prime}, k_{0} x_{0} y\right) \mathrm{d} y \mathrm{~d} t^{\prime \prime} \mathrm{d} t^{\prime}
\end{aligned}
$$

For arbitrary $t$, we can determine the dynamic variation of $\mu_{\varepsilon}$ via numerical experiments. In figure 5 we plot $\mu_{\varepsilon} / \eta_{0}$ as a function of $t$, for $0 \leqslant t \leqslant 400$, for substrate families with $\eta_{0}=10^{-3}$ (solid lines) and $\eta_{0}=5 \times 10^{-4}$ (dashed lines) for two characteristic wavenumbers, $k_{0}=20$ and $k_{0}=40$. We note that the approach towards equilibrium need not be monotonic. For some of these curves we observe a rapid change in $\mu_{\varepsilon}$ for relatively small $t$ prior to relaxation to equilibrium for larger $t$. More importantly, we see that when $k_{0}=20, \mu_{\varepsilon} / \eta_{0}$ approaches a common value as $t \rightarrow \infty$. In figure 5 we also plot the corresponding early-time asymptotics (dash-dotted lines), given by (5.4). 


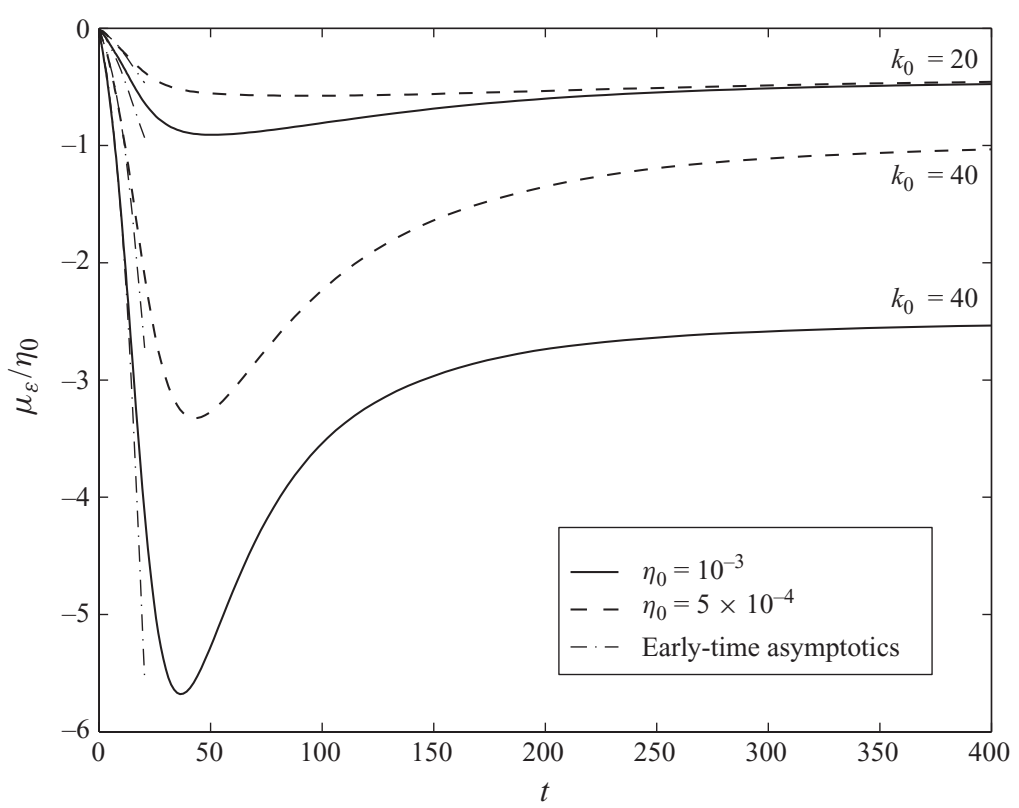

FIGURE 5. Evolution of $\mu_{\varepsilon} / \eta_{0}$ as a function of $t$ for substrate families with $\eta_{0}=10^{-3}$ (solid lines) and $\eta_{0}=5 \times 10^{-4}$ (dashed lines). When $k_{0}=20, \mu_{\varepsilon} / \eta_{0}$ approaches a common value in the long-time limit for both amplitudes.

In the long-time limit, which is crucial in determining the mean of the apparent contact angle at equilibrium, $\left\langle\theta_{a p p}\right\rangle$, we can use the semi-analytical expression for $\left\langle\alpha_{m} \sin k_{m} \ell_{\infty}+\beta_{m} \cos k_{m} \ell_{\infty}\right\rangle$ in (4.9). Conversion of the Riemann sum into an integral yields

$$
\mu_{\varepsilon_{\infty}} \approx \frac{\sqrt{3} \eta_{0}}{2 k_{0}} \int_{0}^{1} G\left(k_{0} y \sqrt{3}\right) F\left(k_{0} y \sqrt{3}\right) \mathrm{d} y,
$$

where as in Part $1, F(\xi)=3 \operatorname{sinc} \xi-3 \cos \xi-\xi \sin \xi$. The above integral can be computed analytically to obtain

$$
\mu_{\varepsilon_{\infty}} \approx-\frac{3 \eta_{0}}{8}\left(2-\cos 2 \sqrt{3} k_{0}\right)-\frac{15 \eta_{0}}{4} \operatorname{sinc} 2 k_{0} \sqrt{3}+\frac{\eta_{0}}{8}\left(17 \sin ^{2} k_{0} \sqrt{3}-6 \operatorname{Cin} 2 k_{0} \sqrt{3}\right) .
$$

In the limit $k_{0} \gg 1, \mu_{\varepsilon_{\infty}}$ has the asymptotic expansion

$$
\mu_{\varepsilon_{\infty}} \sim-\frac{3 \eta_{0}}{8}\left(2-\cos 2 \sqrt{3} k_{0}\right)+O\left(\eta_{0} k_{0}^{-1}\right),
$$

which shows that the mean contact line fluctuation is negative over the regime of interest and also explains the earlier observation in figure 5 that for $k_{0}=20$, the curves for $\mu_{\epsilon} / \eta_{0}$ when $\eta_{0}=10^{-3}$ and $\eta_{0}=5 \times 10^{-4}$ share a common limit. Using (5.1), it can be shown that the ratio of the mean equilibrium apparent contact angle for a droplet on a rough substrate over the apparent contact angle on a flat substrate is given by

$$
\frac{\left\langle\theta_{\text {app }}\right\rangle}{\theta_{\text {flat }}} \sim 1+\frac{\eta_{0} \sqrt{3}}{4}\left(2-\cos 2 \sqrt{3} k_{0}\right)+O\left(\eta_{0} k_{0}^{-1}\right) .
$$

This result suggests that $\left\langle\theta_{a p p}\right\rangle>\theta_{\text {flat }}$, i.e. wetting is ultimately reduced linearly in $\eta_{0}$ over the regime where the perturbation expansion is valid, having also an oscillatory behaviour as $k_{0}$ varies. 


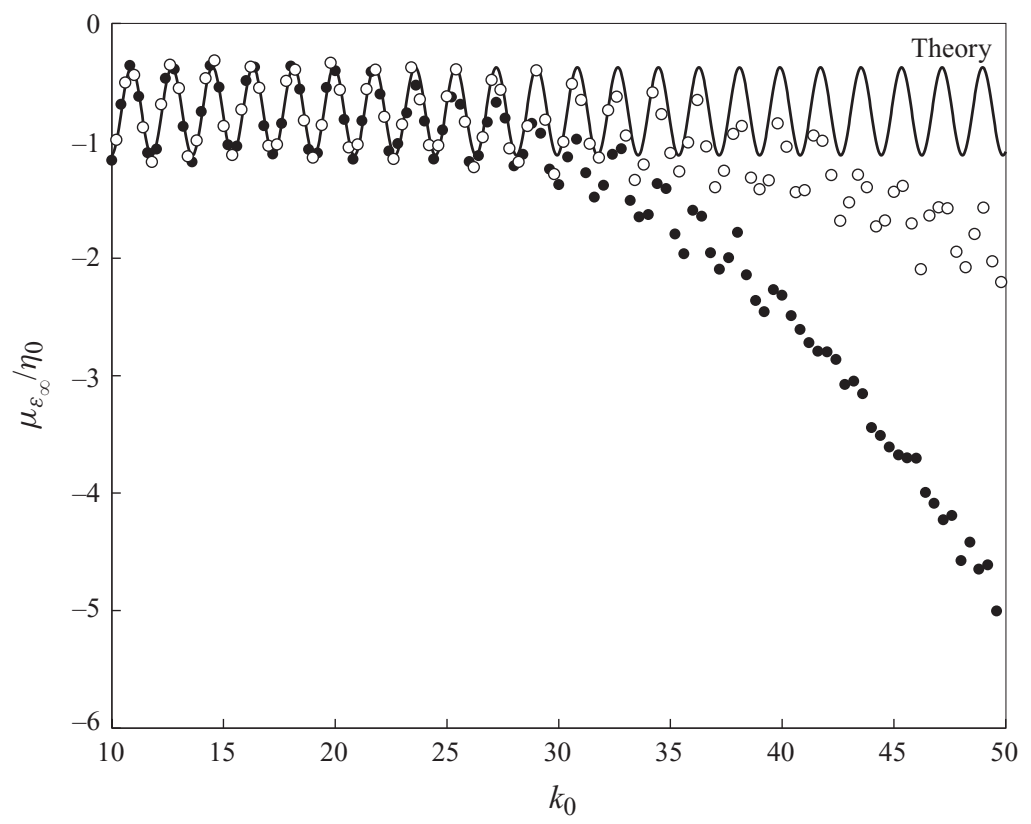

FiguRE 6. Variation of $\mu_{\varepsilon_{\infty}} / \eta_{0}$ as a function of $k_{0}$ for substrate families with $\eta_{0}=5 \times 10^{-4}$ (open circles) and $\eta_{0}=10^{-3}$ (filled circles) together with the corresponding theoretical curve, (5.6). Deviations occur for larger $k_{0}$, as the perturbation condition (3.3) no longer holds.

Figure 6 depicts a plot of (5.6) as a function of $k_{0}$ together with the means obtained from numerical simulations for substrates with various substrate families, with two different $\eta_{0}=10^{-3}$ and $\eta_{0}=5 \times 10^{-4}$. For $k_{0}$ in the region $\sim[10,20]$, the agreement between the semi-analytic approximation and the numerical experiments is excellent, but as the substrate becomes more rough so that $\eta_{0} k_{0}^{2} \ll 1$ is no longer valid, there is a clear deviation towards a progressive reduction of the mean droplet radius. Most importantly, the present analysis demonstrates that, by taking into account the dynamics, substrate roughness ultimately reduces wetting on average. Even though such behaviour appears to contradict Wenzel's law, which predicts wetting enhancement for rough substrates, it signifies the physical fact that the droplet has to overcome the energy barriers that separate the multiple equilibrium droplet states. In other words, in a dynamic setting the droplet can get 'trapped' in an equilibrium state prior to reaching a Wenzel state. This effect is demonstrated in the recent experiments of Chung, Youngblood \& Stafford (2007), where it was found that spreading in a direction perpendicular to the grooves indeed violates Wenzel's law. It is further supported by the work of Cox (1983) on wedge equilibria over three-dimensional rough substrates, who postulated that roughness-induced wetting enhancement is due to a higher-order effect with respect to the substrate amplitude which manifests itself when spreading does not occur in a direction perpendicular to the substrate grooves.

\subsection{Variance of fluctuations}

The variance of $\varepsilon, \sigma_{\varepsilon}^{2}$, is more easily determined for all times compared to its mean, provided that we make some simplifying assumptions. In $\S 4$ we argued that as the number of harmonics increases, $\ell$ becomes less dependent on the values of $\alpha_{m}$ and 


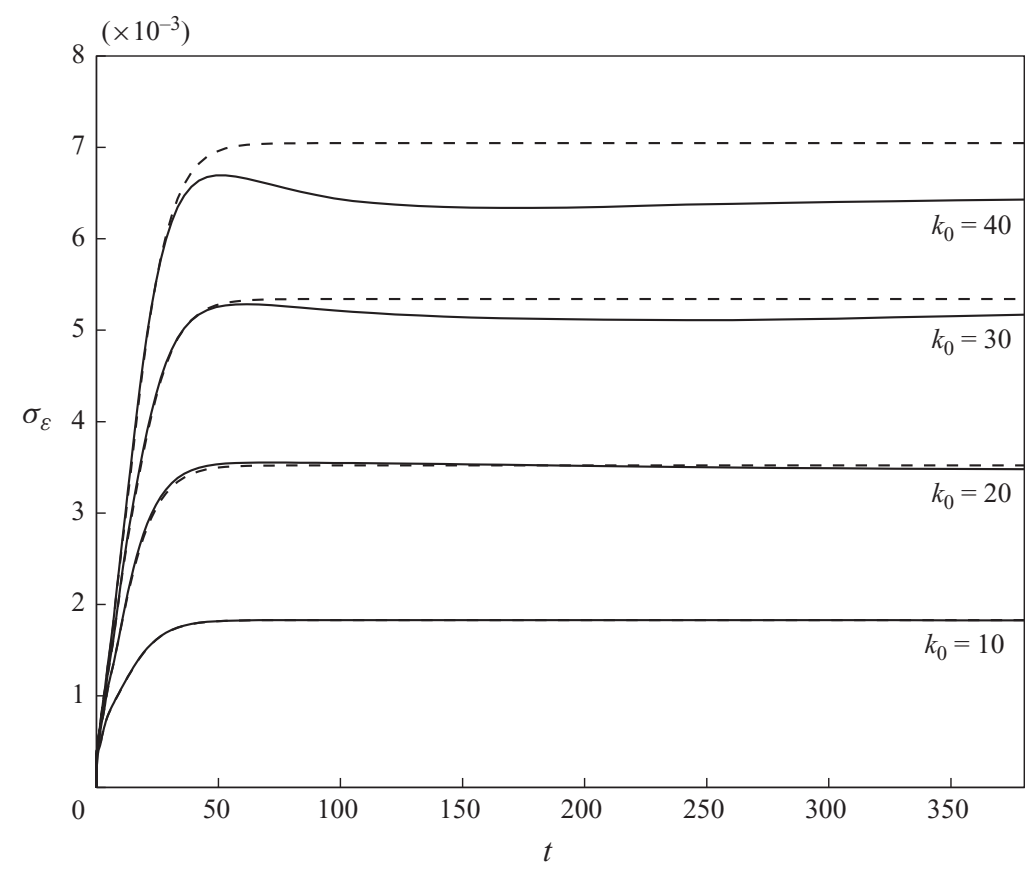

FIGURE 7. Time evolution of $\sigma_{\varepsilon}$ for $\eta_{0}=5 \times 10^{-4}$ and $k_{0}=10,20,30$ and 40 . The solid lines correspond to numerical experiments, whereas the dotted lines to the linear theory (5.10). When $k_{0}=10$, there is perfect agreement between the theory and the numerical experiment.

$\beta_{m}$. Taking this into account when considering the variance of $\varepsilon$, we have

$$
\begin{array}{r}
\sigma_{\varepsilon}^{2} \approx \frac{\eta_{0}^{2}}{N} \sum_{m=1}^{N}\left\langle\alpha_{m}^{2}\right\rangle\left\langle\left[\int_{0}^{t} \sin k_{m} \ell B\left(t^{\prime}, k_{m} x_{0}\right) \exp \left(-\int_{t^{\prime}}^{t} A\left(t^{\prime \prime}\right) \mathrm{d} t^{\prime \prime}\right) \mathrm{d} t^{\prime}\right]^{2}\right\rangle \\
+\left\langle\beta_{m}^{2}\right\rangle\left\langle\left[\int_{0}^{t} \cos k_{m} \ell B\left(t^{\prime}, k_{m} x_{0}\right) \exp \left(-\int_{t^{\prime}}^{t} A\left(t^{\prime \prime}\right) \mathrm{d} t^{\prime \prime}\right) \mathrm{d} t^{\prime}\right]^{2}\right\rangle,
\end{array}
$$

where the cross-terms were neglected on the basis of the mutual independence of the $\alpha_{m}$ and $\beta_{m}$ values. In $\S 4$ we noted that the length scale over which $\ell$ varies is long compared with $\varepsilon$, due to the small characteristic amplitude of the topography. Thus, at least for small $t$, we may take the trigonometric functions of $\ell$ outside the integrals with respect to $t^{\prime}$. Doing so we obtain an approximate expression for $\sigma_{\varepsilon}^{2}$,

$$
\sigma_{\varepsilon}^{2}(t) \approx \eta_{0}^{2} \int_{0}^{1}\left[\int_{0}^{t} B\left(t^{\prime}, y k_{0} x_{0}\right) \exp \left(-\int_{t^{\prime}}^{t} A\left(t^{\prime \prime}\right) \mathrm{d} t^{\prime \prime}\right) \mathrm{d} t^{\prime}\right]^{2} \mathrm{~d} y
$$

where, again, the Riemann sum is converted into an integral as $N \rightarrow \infty$.

Figure 7 shows plots of $\sigma_{\varepsilon}$ as a function of time for $\eta_{0}=5 \times 10^{-4}$ and $k_{0}=10,20,30$ and 40 . We observe that, despite our simplifying assumptions, the agreement between the theoretically predicted curves and those obtained from numerical experiments is quite good, especially for small times. For $k_{0}=10$, the two curves are practically indistinguishable, but as expected the agreement tends to degrade as (3.3) no longer holds. It is worth noting that the time scale over which $\sigma_{\varepsilon}$ saturates is independent of $k_{0}$, compared with $\sigma_{\ell}$ of figure 2 , which is highly dependent on $k_{0}$. Finally, these time 


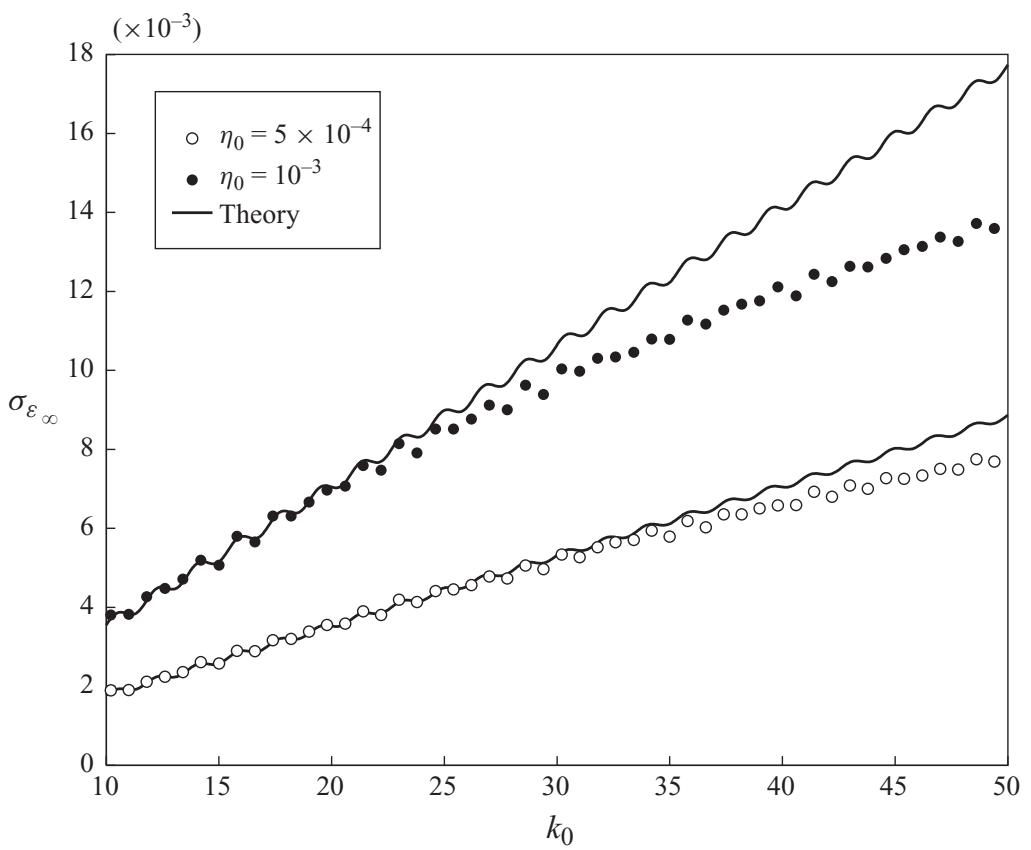

FiguRE 8. Variation of $\sigma_{\varepsilon}$ at equilibrium as a function of $k_{0}$ for substrate families with $\eta_{0}=5 \times 10^{-4}$ (open circles) and $\eta_{0}=10^{-3}$ (filled circles) together with the corresponding theoretical curve (5.11). Agreement degrades as $k_{0}$ increases so that (3.3) is no longer invalid.

scales are much shorter for $\sigma_{\varepsilon}$ compared with $\sigma_{\ell}$, which signifies the fact that when a droplet spreads on a parallel-grooved substrate, it may spend more time sliding along the topographical features than spreading. Thus, the droplet quickly spreads so that its free surface nearly attains its equilibrium shape, but reaching the actual equilibrium takes a longer time due to the sliding motion. Nevertheless, the rate at which this happens is quite small and in reality the slightest bump on the topography can stop the already slowly moving droplet.

In the long-time limit, the variance predicted by (5.10) is identical to (5.3) obtained in Part 1 for the statics, whose asymptotic behaviour is

$$
\sigma_{\varepsilon_{\infty}}^{2} \sim \frac{\eta_{0}^{2} k_{0}^{2}}{8}\left(1-3 \operatorname{sinc} 2 \sqrt{3} k_{0}\right)+O\left(\eta_{0}^{2}\right)
$$

when $k_{0} \gg 1$. The theoretically predicted $\sigma_{\varepsilon_{\infty}}$ is in very good agreement with the standard deviations obtained from numerical simulations as shown in figure 8 , where we plot $\sigma_{\varepsilon_{\infty}}$ as a function of $k_{0}$ when $\eta_{0}=5 \times 10^{-4}$ and $\eta_{0}=10^{-3}$. It is worth noting that as long as (3.3) holds, the leading-order behaviour of $\sigma_{\varepsilon}$ is nearly linear in $\eta_{0} k_{0}$.

\section{Nonlinear effects}

The formalism developed in the previous sections primarily deals with 'weakly' rough substrates so that linearization can be carried out. For substrates that lie beyond the regime of validity of our linearization scheme imposed by the condition (3.3), no analytical prediction can be made and we have to resort to a purely numerical investigation using the full dynamic equations $(2.6 a, b)$. 


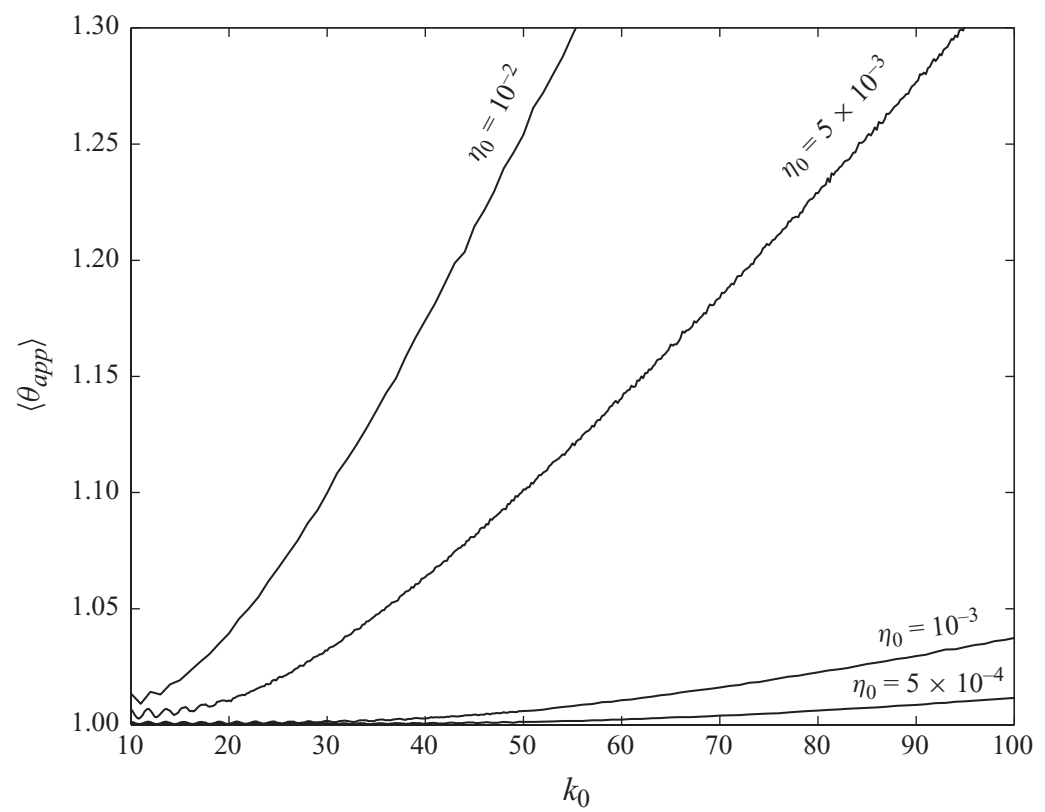

FIGURE 9. Variation of $\left\langle\theta_{a p p}\right\rangle$ as a function of $k_{0}$ for different $\eta_{0}=5 \times 10^{-4}, 10^{-3}, 5 \times 10^{-3}$ and $10^{-2}$. Wetting inhibition increases as the characteristic substrate parameters $\eta_{0}$ and $k_{0}$ increase. The means were computed for discrete values of $k_{0}$, but the lines were joined for the sake of clarity.

As noted earlier, undulations in the substrate topography are expected to impede the motion of the droplet. As these undulations become more frequent (i.e. $k_{0}$ increases), they should naturally inhibit spreading to a greater extent. This holds provided that air is not trapped between the substrate and the droplet. Figure 9 shows plots of $\left\langle\theta_{\text {app }}\right\rangle / \theta_{\text {flat }}$ as a function of $k_{0}$ for different $\eta_{0}$. There is a monotonic increase in the apparent contact angle, which is indicative of wetting inhibition. This substrateinduced hysteresis effect increases with $k_{0}$ and $\eta_{0}$ and for large $k_{0}$, there is a linear increase in the apparent contact angle. Unfortunately our present theory cannot rationalize this behaviour analytically, which then precludes the rigorous derivation of a Wenzel-like formula to describe substrate-induced hysteresis as a function of surface roughness for two-dimensional substrates.

In figure 10 we plot the probability density function of $\theta_{\text {app }}$ at different times for a substrate family with $\eta_{0}=10^{-3}$ and $k_{0}=100$, by solving numerically $(2.6 a, b)$ for $10^{4}$ members of this substrate family. At early times, the probability densities are highly concentrated about the mean, which roughly corresponds to the apparent contact angle when $\eta(x)=0$. At later times, when the effects of the topography are more strongly felt, $\theta_{\text {app }}$ becomes more broadly and less symmetrically distributed about the mean. In the long-time limit, shown in the inset of figure 10, we observe that there can exist substrate realizations that enhance wetting, even though the average behaviour inhibits wetting. By calculating the area of the shaded region, we can infer that there is a finite probability (about $10 \%$ ) for which wetting enhancement occurs, i.e. $\theta_{\text {app }} \leqslant 1$. Consequently, wetting inhibition is meant to be taken in an 'averaged sense', since not all realizations may exhibit such behaviour.

Wetting inhibition occurs not only for advancing fronts but also for receding ones. To illustrate this, we performed numerical experiments over $10^{4}$ substrate realizations 


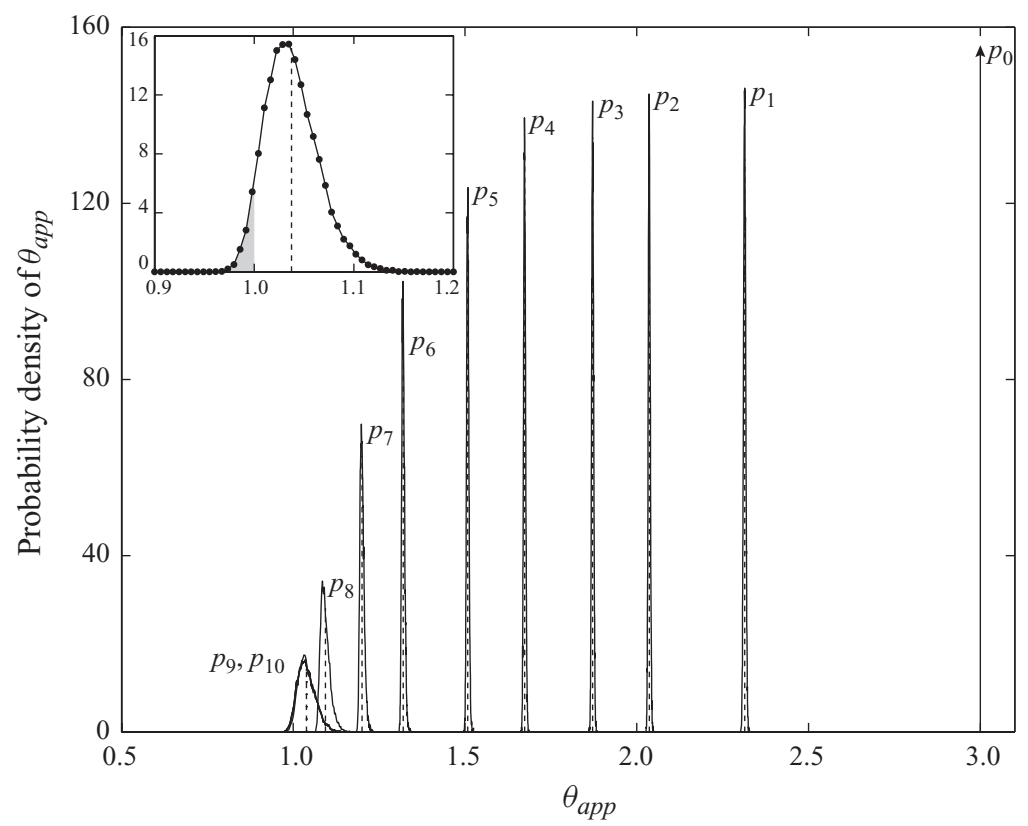

Figure 10. Evolution of the probability density of $\theta_{a p p}$ when $\eta_{0}=10^{-3}$ and $k_{0}=100$. The densities $p_{0}, p_{1}, \ldots, p_{10}$ correspond to times $t=0,0.2,0.4,0.6,1,1.5,3,5,10,50$ and 500, respectively. At $t=0$, we assume a deterministic apparent contact angle $\theta_{a p p}=3$. The discrete points of each probability density have been joined for clarity and the dashed curves show the location of the mean. Inset: the probability density of $\theta_{a p p}$ in the long-time limit. The shaded region indicates that a substrate realization may enhance wetting with finite probability.

by solving numerically $(2.6 a, b)$ for droplet fronts that both initially advance, $a(0)=-b(0)=1$ and recede, $a(0)=-b(0)=2$. As noted by Savva \& Kalliadasis (2009), a droplet front may not exhibit a single behaviour (i.e. either advancing or receding) for all times, but can both advance and recede during its motion, especially when the droplet is close to its equilibrium. The results of our computations are shown in figure 11, where we plot $\theta_{a p p}$ as a function of the rate of change of the droplet radius, $(\dot{a}-\dot{b}) / 2$. Because of the substrate-induced hysteresis effect, it is generally observed that the apparent contact angle at equilibrium for advancing fronts, $\theta_{a}$, is different from the apparent contact angle at equilibrium for receding ones, $\theta_{r}$. In this particular case we have that on average $\theta_{a}-\theta_{r} \approx 0.08$. As we show in figure 11 , an ensemble average at each time step yields a smooth, one-to-one, curve (solid line) compared with a more oscillatory curve about the mean for a particular substrate realization (dashed line). During such oscillations, the moving fronts usually exhibit stick-slip behaviour.

Stick-slip dynamics is difficult to characterize because there can exist a wide variability on how and when stick-slip events occur. Using the same computations as those of figure 10, we depict in figure 12 some representative cases where stick-slip occurs. Low-amplitude heterogeneities do not significantly affect the moving fronts at the onset of spreading, since in all cases presented in the figure, the evolution is nearly indistinguishable from the flat-substrate case for small $t$. Different stick-slip behaviours occur, however, as the droplet approaches equilibrium and its speed is low enough so that the topographical variations become important. For example, in figure $12(a)$ the left contact point (l.c.p.) gets pinned whereas the right one (r.c.p.) 


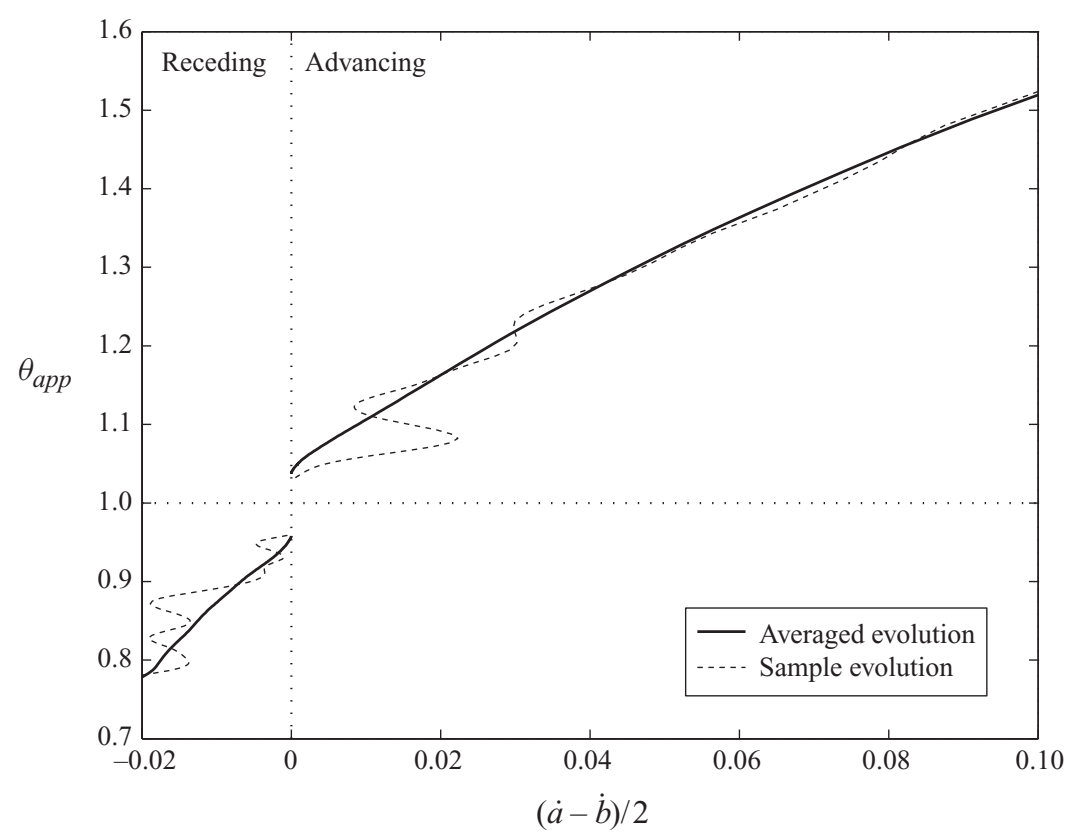

FIGURE 11. Apparent contact angle as a function of the rate of change of the droplet radius, illustrating substrate-induced contact angle hysteresis. For each numerical experiment, the droplet lies initially either at $a(0)=-b(0)=1$ (advancing) or at $a(0)=-b(0)=2$ (receding). The solid line corresponds to the curve obtained for an ensemble average taken for fixed $t$ over $10^{4}$ different substrates. The dashed line shows a sample evolution curve for a particular realization.

continues to move in a series of weak pinning, de-pinning events. In figure 12(b), both fronts appear to be stuck for some time before the r.c.p. depins. Stick-slip events commonly occur for one of the contact points. However, de-pinning can happen for both the r.c.p. and 1.c.p. as figure $12(c)$ indicates. It is also possible for multiple stick-slip events to occur for a single front (see figure 12d). Figure 12(e) shows that stick-slip can occur even at long times and figure $12(f)$ shows that consecutive depinning events can happen close to each other. Figure $12(g, h)$ shows the corresponding spreading rates for figure 12(e,f). Despite the remarkable similarity in the evolution of the spreading rates, the relatively minor differences lead to a markedly different evolution for the two moving fronts.

\section{Generalization to arbitrary substrate representations}

Thus far, we have almost exclusively focused on substrate families described by band-limited white-noise spectral densities as a means to illustrate the concepts we have developed throughout this study. As we have pointed out in Part 1, one of the primary reasons for choosing such substrates is that they are completely prescribed by two parameters, $\eta_{0}$ and $k_{0}$. Moreover, we demonstrated in $\S 6$ of Part 1 that such substrates are able to adequately capture qualitatively the leading-order behaviour of the statistical properties of the two fronts, as the statistics do not change with respect to $\eta_{0}$ and $k_{0}$, apart from a numerical prefactor that depends on the specific form of the spectral density of the substrate. 
(a)

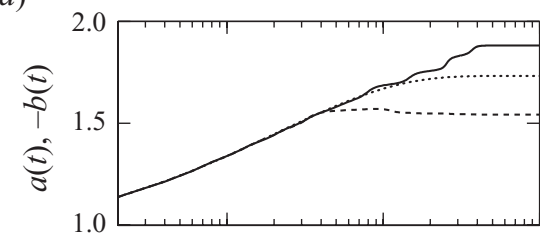

(c)

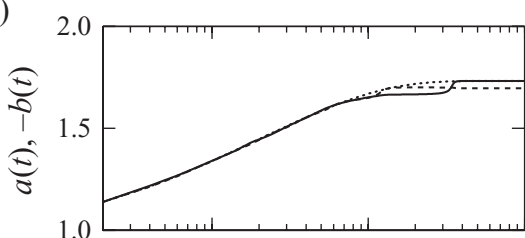

(e)

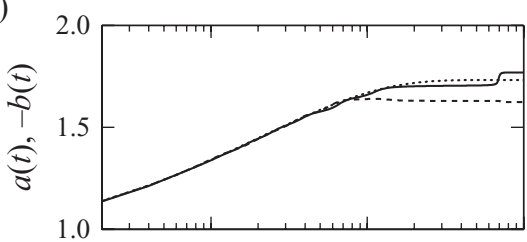

$(g)$

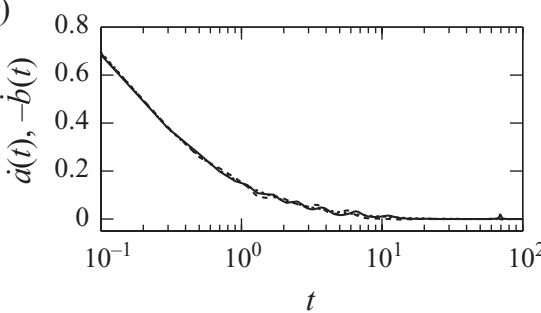

(b)

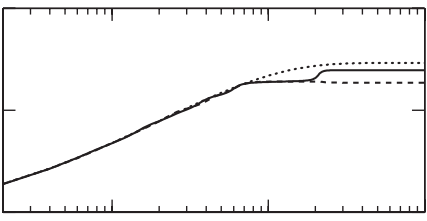

(d)

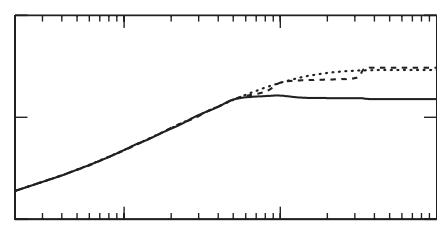

$(f)$

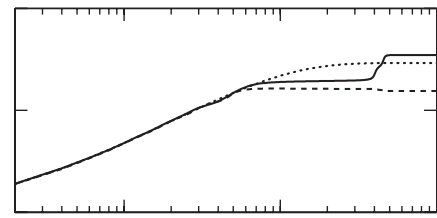

(h)

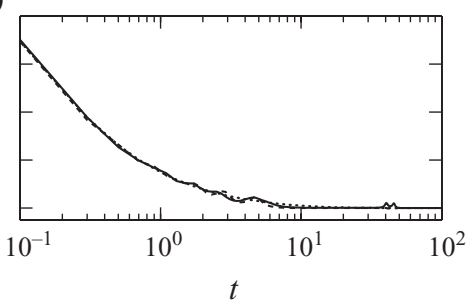

FIGURE 12. Demonstration of pinning-de-pinning dynamics from numerical experiments using random realizations of (2.1). Plots $(a-f)$ show the evolution curves of $a(t)$ (r.c.p. solid lines) and $-b(t)$ (1.c.p. dashed lines), illustrating different pinning/de-pinning scenarios. At the onset, all droplets are oblivious to the substrate variations and the evolution curves nearly follow the flat-substrate evolution curve (dotted line). Topography effects become important when the droplet spreads sufficiently slowly. Plots $(g)$ and $(h)$ show the spreading rates for plots $(e)$ and $(f)$, respectively. The final location of the droplet is markedly different regardless of the fact that the spreading rates resemble each other.

In this section, we will go a step further, by presenting an analysis that facilitates the extraction of the leading-order statistics of wetting, without knowing the precise form of the spectral density. To achieve this, it is necessary to define another set of length scales, which will yield expressions that are able to fully capture the leadingorder statistics, allowing us to go beyond the qualitative agreement we were able to achieve previously. Naturally, to properly quantify the statistics, these new length scales should encompass information from the spectral density of the substrate family. Apart from the intricacies encountered in the analysis that will follow, it is also of interest from a more practical point of view, as it is generally much more difficult to measure the spectral density of a substrate experimentally, compared to some length scale that arises in a natural fashion and can be trivially extracted from substrate profilometry measurements.

Since wetting is essentially characterized by the statistics of $\varepsilon$ at a stable equilibrium, we will only focus on $\varepsilon$, no longer requiring for $\ell$ to be the closest equilibrium to 0 , as 
in zero. Physically, this amounts to looking at the statistics of the equilibria attained by a droplet that is randomly placed on the substrate. Contrary to the analysis of Part 1, we now take into account the stability criterion derived in $\S 4$, aiming to establish more rigorously the wetting inhibition for all substrates with band-limited spectral densities, which was earlier observed for band-limited white noise substrates.

Hence, the starting point is to use a substrate family with arbitrary spectral density $S(k)$, given by

$$
\eta(x)=\sum_{m=1}^{+\infty}\left(\alpha_{m} \sin k_{m} x+\beta_{m} \cos k_{m} x\right) \sqrt{S\left(k_{m}\right) \Delta k},
$$

where $k_{m}-k_{m-1}=\Delta k$ and $k_{1}=0$, aiming to deduce the statistics of

$$
\varepsilon=\frac{1}{2} \sum_{m=1}^{+\infty}\left(\alpha_{m} \sin k_{m} \ell+\beta_{m} \cos k_{m} \ell\right) F\left(k_{m} \sqrt{3}\right) \sqrt{S\left(k_{m}\right) \Delta k},
$$

which is the same as $(6.3 a)$ of Part 1 . Here, $\ell$ is not arbitrary and needs to satisfy the additional constraints:

$$
\begin{gathered}
\sum_{m=1}^{+\infty}\left(\alpha_{m} \cos k_{m} \ell-\beta_{m} \sin k_{m} \ell\right) G\left(k_{m} \sqrt{3}\right) \sqrt{S\left(k_{m}\right) \Delta k}=0, \\
\sum_{m=1}^{+\infty} k_{m}\left(\alpha_{m} \sin k_{m} \ell+\beta_{m} \cos k_{m} \ell\right) G\left(k_{m} \sqrt{3}\right) \sqrt{S\left(k_{m}\right) \Delta k}>0 .
\end{gathered}
$$

The first equation above is the same as $(6.3 b)$ of Part 1 and the second one is the generalization of the stability criterion, given by (4.7). We readily see that the $\ell$ values that satisfy (7.3) give the locations of the maxima of

$$
Q(\ell)=\sum_{m=1}^{+\infty}\left(\alpha_{m} \sin k_{m} \ell+\beta_{m} \cos k_{m} \ell\right) k_{m}^{-1} G\left(k_{m} \sqrt{3}\right) \sqrt{S\left(k_{m}\right) \Delta k} .
$$

Analytical progress can be made by appropriately casting this problem as one that bears similarities with a classical problem in probability theory, that of determining the statistics of the local maxima of a stationary random function. This problem has been investigated in the work of Rice (1939), where he obtained the distribution of local maxima for random functions such as the one in (7.4). More specifically, for the local maxima of $Q(\ell)$, he found that they are distributed according to the probability density $P_{Q_{\max }}$, given by

$$
P_{Q_{\max }}=-n_{Q}^{-1} \int_{-\infty}^{0} \zeta \tilde{p}(Q, 0, \zeta) \mathrm{d} \zeta
$$

where $n_{Q}$ is the number of maxima of $Q$ per unit length and $\tilde{p}\left(Q, Q^{\prime}, Q^{\prime \prime}\right)$ is the joint probability density function of the Gaussian random variables $Q, Q^{\prime}$ and $Q^{\prime \prime}$, where the primes denote differentiation of a function with respect to its argument (in this case $\ell$ ). Here we are interested in the statistics of the additional equation (7.2), which is of course not present in Rice's original analysis. Consequently, the problem is to find the statistics of $\varepsilon$, provided that $\ell$ locally maximize $Q(\ell)$.

Since $\varepsilon$ is correlated with $Q$ and its derivatives, we consider their joint probability density function, $p\left(Q, Q^{\prime}, Q^{\prime \prime}, \varepsilon\right)$, given in terms of $\boldsymbol{x}=\left[Q, Q^{\prime}, Q^{\prime \prime}, \varepsilon\right]^{\mathrm{T}}$ and the square 
matrix composed of the second moments of the $\boldsymbol{x}$ values for fixed $\ell, \boldsymbol{M}$,

$$
p\left(Q, Q^{\prime}, Q^{\prime \prime}, \varepsilon\right)=\frac{1}{4 \pi^{2} \sqrt{\|\boldsymbol{M}\|}} \exp \left(-\frac{1}{2} \boldsymbol{x}^{\mathrm{T}} \boldsymbol{M}^{-1} \boldsymbol{x}\right),
$$

where $\|\boldsymbol{M}\|$ and $\boldsymbol{M}^{-1}$ represent the determinant and inverse of $\boldsymbol{M}$, respectively (Cramér 1962). The entries of $\boldsymbol{M}$ are

$$
\boldsymbol{M}=\left[\begin{array}{cccc}
\left\langle Q^{2}\right\rangle_{\ell} & 0 & \left\langle Q Q^{\prime \prime}\right\rangle_{\ell} & \langle Q \varepsilon\rangle_{\ell} \\
0 & \left\langle Q^{\prime 2}\right\rangle_{\ell} & 0 & 0 \\
\left\langle Q Q^{\prime \prime}\right\rangle_{\ell} & 0 & \left\langle Q^{\prime \prime 2}\right\rangle_{\ell} & \left\langle Q^{\prime \prime} \varepsilon\right\rangle_{\ell} \\
\langle Q \varepsilon\rangle_{\ell} & 0 & \left\langle Q^{\prime \prime} \varepsilon\right\rangle_{\ell} & \left\langle\varepsilon^{2}\right\rangle_{\ell}
\end{array}\right],
$$

where $\langle\cdot\rangle_{\ell}$ denotes an ensemble average for fixed $\ell$. In this general formulation, the probability density function of $\varepsilon$ is given by

$$
P(\varepsilon)=-n_{Q}^{-1} \int_{-\infty}^{+\infty} \int_{-\infty}^{0} \zeta p(\xi, 0, \zeta, \varepsilon) \mathrm{d} \zeta \mathrm{d} \xi,
$$

which comes from (7.5) and an integration over all attainable values for $Q$. In terms of the entries of $\mathrm{M}, n_{Q}$ is given by (Rice 1945)

$$
n_{Q}=\frac{1}{2 \pi} \sqrt{\frac{\left\langle Q^{\prime \prime 2}\right\rangle_{\ell}}{\left\langle Q^{\prime 2}\right\rangle_{\ell}}}
$$

The integral in (7.8) may be computed analytically so that $P(\varepsilon)$ is expressed as the superposition of the probability density of a zero-mean normal variable together with a smaller, perturbative term that is responsible for the non-zero mean. This establishes rigorously our earlier argument where we took $\varepsilon$ to be a normal variable to leading order. This deviation from normality is merely a consequence of the fact that $\varepsilon$ is expressed as an infinite sum of weakly dependent random variables, whereas the central limit theorem is expected to exactly hold provided that the random variables are mutually independent.

Nevertheless, our principal interest is to determine $\langle\varepsilon\rangle$ and $\left\langle\varepsilon^{2}\right\rangle$, since we have already found that $\varepsilon$ is nearly a normal variable. From the probability density function of $\varepsilon$, we can extract, after some rather lengthy algebra, analytical expressions for the first two moments of $\varepsilon$, given as

$$
\begin{aligned}
\langle\varepsilon\rangle & =-\left\langle Q^{\prime \prime} \varepsilon\right\rangle_{\ell} \sqrt{\frac{\pi}{2\left\langle Q^{\prime \prime 2}\right\rangle_{\ell}}}, \\
\left\langle\varepsilon^{2}\right\rangle & =\left\langle\varepsilon^{2}\right\rangle_{\ell}+\frac{\left\langle Q^{\prime \prime} \varepsilon\right\rangle_{\ell}^{2}}{\left\langle Q^{\prime \prime 2}\right\rangle_{\ell}} .
\end{aligned}
$$

The expressions above provide information about the statistics of $\varepsilon$ in the most general form. However, as we ideally want to investigate the 'roughest' substrates permitted by the perturbation condition (3.3), we will proceed by obtaining the leading-order expressions with respect to some large scale, taken to be an upper cutoff wavenumber $k_{c}$, for which $S(k)=0$ for $k>k_{c}$. The second moments, $\left\langle\varepsilon^{2}\right\rangle_{\ell}$, $\left\langle Q^{\prime \prime 2}\right\rangle_{\ell}$ and $\left\langle Q^{\prime \prime} \varepsilon\right\rangle_{\ell}$, can be expressed in terms of the autocovariance function of $\eta(x)$ and its derivatives, given by

$$
\psi_{\tau}=\langle\eta(x) \eta(x+\tau)\rangle=\int_{0}^{+\infty} S(k) \cos k \tau \mathrm{d} k .
$$


In particular, for 'rough' substrates, the most important contributions come from the first two terms from each of the following:

$$
\begin{aligned}
\left\langle\varepsilon^{2}\right\rangle_{\ell} & \sim-\frac{3}{8} \psi_{0}^{\prime \prime}-\frac{3}{8} \int_{0}^{k_{c}} k^{2} S(k) \cos 2 k \sqrt{3} \mathrm{~d} k, \\
\left\langle Q^{\prime \prime 2}\right\rangle_{\ell} & \sim \frac{3}{2} \psi_{0}^{\prime \prime \prime \prime}+\frac{3}{2} \int_{0}^{k_{c}} k^{4} S(k) \cos 2 k \sqrt{3} \mathrm{~d} k, \\
\left\langle\varepsilon Q^{\prime \prime}\right\rangle_{\ell} & \sim-\frac{\sqrt{3}}{2} \psi_{0}^{\prime \prime}+\frac{3}{4} \int_{0}^{k_{c}} k^{3} S(k) \sin 2 k \sqrt{3} \mathrm{~d} k
\end{aligned}
$$

Contributions due to a lower cutoff wavenumber, say $k_{l}$, are neglected on the basis of the assumption that $k_{c} \gg k_{l}$. Furthermore, with the most generality, the spectral density is taken to have $m_{c} \in \mathbb{N}_{0}=\{0,1,2, \ldots\}$ vanishing derivatives at $k=k_{c}$ so that near $k_{c}$ it behaves like

$$
S(k) \sim \frac{S_{c}}{m_{c} !}\left(k-k_{c}\right)^{m_{c}}+O\left(\left(k-k_{c}\right)^{m_{c}+1}\right),
$$

where $S_{c}$ is some constant. Hence, we have that, for $m_{c}=2 n, n \in \mathbb{N}_{0}$,

$$
\begin{aligned}
\left\langle\varepsilon^{2}\right\rangle_{\ell} & \sim-\frac{3}{8} \psi_{0}^{\prime \prime}-\frac{3}{8}\left(-\frac{1}{12}\right)^{n} k_{c}^{3} S_{c} \operatorname{sinc} 2 k_{c} \sqrt{3} \\
\left\langle Q^{\prime \prime 2}\right\rangle_{\ell} & \sim \frac{3}{2} \psi_{0}^{\prime \prime \prime \prime}+\frac{3}{2}\left(-\frac{1}{12}\right)^{n} k_{c}^{5} S_{c} \operatorname{sinc} 2 k_{c} \sqrt{3} \\
\left\langle Q^{\prime \prime} \varepsilon\right\rangle_{\ell} & \sim-\frac{\sqrt{3}}{2} \psi_{0}^{\prime \prime}+\frac{3 \sqrt{3}}{2}\left(-\frac{1}{12}\right)^{n+1} k_{c}^{3} S_{c} \cos 2 k_{c} \sqrt{3}
\end{aligned}
$$

and for $m_{c}=2 n+1$ that

$$
\begin{aligned}
\left\langle\varepsilon^{2}\right\rangle_{\ell} & \sim-\frac{3}{8} \psi_{0}^{\prime \prime}-\frac{3}{8}\left(-\frac{1}{12}\right)^{n+2} k_{c}^{2} S_{c} \cos 2 k_{c} \sqrt{3}, \\
\left\langle Q^{\prime \prime 2}\right\rangle_{\ell} & \sim \frac{3}{2} \psi_{0}^{\prime \prime \prime \prime}+\frac{3}{2}\left(-\frac{1}{12}\right)^{n+2} k_{c}^{4} S_{c} \cos 2 k_{c} \sqrt{3}, \\
\left\langle Q^{\prime \prime} \varepsilon\right\rangle_{\ell} & \sim-\frac{\sqrt{3}}{2} \psi_{0}^{\prime \prime}-\frac{3 \sqrt{3}}{2}\left(-\frac{1}{12}\right)^{n+1} k_{c}^{4} S_{c} \operatorname{sinc} 2 k_{c} \sqrt{3}
\end{aligned}
$$

For the second moment of $\varepsilon$, one may verify that $\left\langle\varepsilon^{2}\right\rangle_{\ell} \gg\left\langle Q^{\prime \prime} \varepsilon\right\rangle_{\ell} /\left\langle Q^{\prime \prime 2}\right\rangle_{\ell}$ and hence $\left\langle\varepsilon^{2}\right\rangle \sim\left\langle\varepsilon^{2}\right\rangle_{\ell}$. It is noteworthy that this analysis proves a result we have previously obtained in Part 1 using less rigorous arguments. Hence,

$$
\left\langle\varepsilon^{2}\right\rangle \sim \begin{cases}-\frac{3}{8} \psi_{0}^{\prime \prime}-\frac{3}{8}\left(-\frac{1}{12}\right)^{n} k_{c}^{3} S_{c} \operatorname{sinc} 2 k_{c} \sqrt{3}, & m_{c}=2 n, \\ -\frac{3}{8} \psi_{0}^{\prime \prime}-\frac{3}{8}\left(-\frac{1}{12}\right)^{n+2} k_{c}^{2} S_{c} \cos 2 k_{c} \sqrt{3}, & m_{c}=2 n+1,\end{cases}
$$


where the contributions due to $\psi_{0}^{\prime \prime}$ are the highest. For the mean of $\varepsilon$, we have

$$
\langle\varepsilon\rangle \sim \begin{cases}\sqrt{\frac{\pi}{4 \psi_{0}^{\prime \prime \prime \prime}}}\left[\psi_{0}^{\prime \prime}-3\left(-\frac{1}{12}\right)^{n+1} k_{c}^{3} S_{c} \cos 2 k_{c} \sqrt{3}\right], & m_{c}=2 n, \\ \sqrt{\frac{\pi}{4 \psi_{0}^{\prime \prime \prime \prime}}}\left[\psi_{0}^{\prime \prime}+3\left(-\frac{1}{12}\right)^{n+1} k_{c}^{4} S_{c} \operatorname{sinc} 2 k_{c} \sqrt{3}\right], & m_{c}=2 n+1,\end{cases}
$$

where both terms are now of the same order. Here $\langle\varepsilon\rangle$ and $\left\langle\varepsilon^{2}\right\rangle$ depend on integrals of the spectral density function, $\psi_{0}^{\prime \prime}$ and $\psi_{0}^{\prime \prime \prime \prime}$. Interestingly, knowledge of the number of maxima per unit length, $n_{\max }$, and their mean, $\bar{\eta}_{\max }$, suffice to determine $\psi_{0}^{\prime \prime}$ and $\psi_{0}^{\prime \prime \prime \prime}$. Consequently, it is not necessary to know $S(k)$ everywhere to compute these integrals, since all the required information is already contained in these two length scales. Hence, we have that (Rice 1945)

$$
n_{\max }=\frac{1}{2 \pi} \sqrt{-\frac{\psi_{0}^{\prime \prime \prime \prime}}{\psi_{0}^{\prime \prime}}} \quad \text { and } \quad \bar{\eta}_{\max }=-\psi_{0}^{\prime \prime} \sqrt{\frac{\pi}{2 \psi_{0}^{\prime \prime \prime \prime}}} .
$$

Solving for $\psi_{0}^{\prime \prime}$ and $\psi_{0}^{\prime \prime \prime \prime}$ yields

$$
\begin{aligned}
\psi_{0}^{\prime \prime} & =-8 \pi n_{\text {max }}^{2} \bar{\eta}_{\text {max }}^{2}, \\
\psi_{0}^{\prime \prime \prime \prime} & =32 \pi^{3} n_{\max }^{4} \bar{\eta}_{\text {max }}^{2},
\end{aligned}
$$

and therefore

$$
\begin{gathered}
\left\langle\varepsilon^{2}\right\rangle \sim\left\{\begin{array}{l}
3 \pi n_{\text {max }}^{2} \bar{\eta}_{\text {max }}^{2}-\frac{3}{8}\left(-\frac{1}{12}\right)^{n} k_{c}^{3} S_{c} \operatorname{sinc} 2 k_{c} \sqrt{3}, \quad m_{c}=2 n, \\
3 \pi n_{\text {max }}^{2} \bar{\eta}_{\text {max }}^{2}-\frac{3}{8}\left(-\frac{1}{12}\right)^{n+2} k_{c}^{2} S_{c} \cos 2 k_{c} \sqrt{3}, \quad m_{c}=2 n+1,
\end{array}\right. \\
\langle\varepsilon\rangle \sim \begin{cases}-\frac{\bar{\eta}_{\max }}{\sqrt{2}}\left[1-\frac{k_{c}^{3} S_{c} \cos 2 k_{c} \sqrt{3}}{32 \pi n_{\max }^{2} \bar{\eta}_{\max }^{2}(-12)^{n}}\right], & m_{c}=2 n, \\
-\frac{\bar{\eta}_{\max }}{\sqrt{2}}\left[1+\frac{k_{c}^{4} S_{c} \operatorname{sinc} 2 k_{c} \sqrt{3}}{32 \pi n_{\max }^{2} \bar{\eta}_{\max }^{2}(-12)^{n}}\right], & m_{c}=2 n+1 .\end{cases}
\end{gathered}
$$

In addition to the statistics of $\varepsilon$, knowing $\psi_{0}^{\prime \prime}$ allows us to obtain an expression for the roughness coefficient, $r$, defined in (2.4) of Part 1, which is given by

$$
r \approx 1-\frac{1}{2} \psi_{0}^{\prime \prime}=1+4 \pi n_{\max }^{2} \bar{\eta}_{\max }^{2}
$$

in its more general form. Through the analysis we presented above, we were able to obtain the leading-order statistics of the droplet radius at a stable equilibrium in a form that is independent of the spectral density of the substrate we employ, compared with the analysis presented in $\S 6$ of Part 1 , where the form of the results we obtained was highly dependent on the specifics of $S(k)$. On the other hand, here we found that provided that the appropriate length scales are used, namely $n_{\max }$ and $\bar{\eta}_{\max }$, the leading-order expression for the second moment for $\varepsilon$ is always $\left\langle\varepsilon^{2}\right\rangle \sim 3 \pi n_{\max }^{2} \bar{\eta}_{\max }^{2}$ irrespective of the precise form for $S(k)$, because these two scales already contain information from the spectral density. The two length scales given by (7.25) are easily determined from an experimental substrate profile; determining the next-order term however requires additional information about the behaviour of $S(k)$ near the cutoff, 
$k_{c}$. Hence, $n_{\max }$ and $\bar{\eta}_{\max }$ may be regarded, respectively, as the natural characteristic wavenumber and amplitude for the substrate family, whereas $k_{0}$ and $\eta_{0}$ appear to be more mathematically than physically motivated.

To make the connection with the band-limited white noise used throughout this study for which

$$
S(k)= \begin{cases}\frac{\eta_{0}^{2}}{k_{0}}, & 0 \leqslant k \leqslant k_{c}, \\ 0, & k>k_{c} .\end{cases}
$$

In this case, we have the parameters $m_{c}=0, k_{c}=k_{0}, S_{c}=\eta_{0}^{2} / k_{0}, n_{\max }=k_{0} \sqrt{3 / 5} /(2 \pi)$ and $\bar{\eta}_{\max }=\eta_{0} \sqrt{10 \pi} / 6$. Using (7.28) and (7.29), we obtain

$$
\begin{aligned}
\left\langle\varepsilon^{2}\right\rangle & \sim \frac{1}{8} \eta_{0}^{2} k_{0}^{2}\left(1-3 \operatorname{sinc} 2 k_{0} \sqrt{3}\right), \\
\langle\varepsilon\rangle & \sim-\frac{1}{6} \eta_{0} \sqrt{5 \pi}\left(1-\frac{3}{4} \cos 2 k_{0} \sqrt{3}\right) .
\end{aligned}
$$

The first equation corresponds exactly to the variance of $\varepsilon$ obtained earlier, given also that $\left\langle\varepsilon^{2}\right\rangle \gg\langle\varepsilon\rangle^{2}$. The mean of $\varepsilon$, (7.33) differs from the semi-analytical expression (5.7), which was verified by our numerical experiments. The difference between the two expressions is due to the way the problem was posed. To get (5.7), $\ell$ is treated as a solution to a first-passage problem by assuming initial symmetry about the $x$-axis such that $\ell(0)=0$, whereas (7.32) takes into account all possible stable equilibria.

\section{Conclusions}

We have analysed the dynamics of a two-dimensional droplet spreading over randomly varying shallow substrates in the limit of small contact angles, building upon the theoretical framework introduced in Part 1 and the results from the recent work of Savva \& Kalliadasis (2009) on droplet spreading over deterministic substrates. We obtained a set of differential equations for the time evolution of the droplet shift, $\ell$, and the contact line fluctuations, $\varepsilon$, which correspond to the sliding and spreading components of the droplet motion, respectively. These equations cannot be treated with the standard Langevin-Fokker-Planck formalism of stochastic dynamics and special statistical approaches for their solution had to be developed.

The droplet shift, which is independent of the contact line fluctuations, is governed by a nonlinear differential equation which can only be solved numerically for arbitrary times. However, the early-time and the long-time behaviours of the droplet shift can be obtained analytically. In the long-time limit, in particular, we found its variance to scale like $\operatorname{Var}[\ell] \sim O\left(k_{0}^{-2}\right)$, which is independent of the characteristic amplitude, $\eta_{0}$. On the other hand, the contact line fluctuation is governed by a linear differential equation, and is therefore predicted to be a normally distributed normal variable for all times. Some simplifying assumptions allowed us to deduce the leading-order variance for all times; in the long-time limit in particular, $\operatorname{Var}[\varepsilon] \sim O\left(\eta_{0}^{2} k_{0}^{2}\right)$.

Obtaining the time evolution of the mean of the contact line fluctuation is a substantially more difficult task due to the dependence of the $\varepsilon$-dynamics on $\ell$. However, we were able to deduce the long-time behaviour for the mean, which predicts wetting inhibition on average, thus allowing us to fully assess the influence of substrate roughness on the wetting characteristics. Our theoretical predictions are in excellent agreement with numerical experiments in a regime where $\eta_{0} k_{0}^{2} \ll 1$. We 
also demonstrated a number of intriguing features. In particular, by examining the evolution of $\varepsilon$ and $\ell$ we showed the tendency of the droplet to slide along the substrate without spreading before reaching equilibrium, the presence of a stick-slip behaviour that is rather sensitive to the substrate features and the static contact angle and a substrate-induced, hysteresis-like effect. Finally, our analysis was generalized to arbitrary substrate representations. We showed, in particular, that the statistics depend on naturally occurring length scales such as the mean number of substrate maxima per unit length and their mean value.

To summarize this two-part study, we have presented a detailed and systematic investigation of the motion of a droplet over randomly and slowly varying substrates in the limit of small contact angles that is based solely on hydrodynamic principles. In Part 1, we modelled the substrates as stationary random functions and investigated the linearized droplet equilibria on such substrates. We focused mostly on substrates represented by band-limited white-noise spectral densities, mostly because such densities were completely specified by only two length scales that are readily available from experiments, but we found that there are no qualitative changes had the spectral density been different. We also found that $\varepsilon$ is nearly normally distributed and the statistics of $\ell$ are most appropriately studied as a first-passage problem, which could only be solved approximately via Padé approximants. Most importantly, we concluded that the effects of substrate roughness on wetting cannot be assessed by statics alone, since such analysis is carried out irrespective of the stability of the equilibria considered. In Part 2 we focused on the dynamics, where we considered a set of evolution equations for the linearized dynamics in the limit of shallow substrates, obtaining the statistics of $\varepsilon$ and $\ell$ both in the early- and long-time limits of spreading. Aside from our main results we already summarized above in this section, it is important to reiterate that it is only by studying the dynamics one can correctly predict the effects on wetting. One of our main findings is that wetting inhibition can be observed in two dimensions, which appears to increase with substrate roughness. Also noteworthy is our generalization to arbitrary spectral densities, which allows us to identify the proper, natural length scales of the problem, which may potentially facilitate direct comparisons of the droplet behaviour among substrates belonging to families with different spectral densities.

Although the results of the present study are restricted to two dimensions, thus avoiding some of the intricacies of spreading in three dimensions, they nevertheless show that taking into consideration the details of spreading dynamics through a hydrodynamic model obtained from first principles as well as the way by which droplet equilibria are approached in time, it is crucial in analysing the effects of substrate roughness on wetting.

We acknowledge financial support from EPSRC Platform Grant No. EP/E046029 and ERC Advanced Grant No. 247031.

\section{REFERENCES}

Abramowitz, M. \& Stegun, I. A. 1964 Handbook of Mathematical Functions with Formulas, Graphs, and Mathematical Tables. Dover.

Breiman, L. 1992 Probability. SIAM.

Cazabat, A. M. \& Cohen-Stuart, M. A. 1986 Dynamics of wetting: effects of surface roughness. J. Phys. Chem. 90, 5845-5849.

Chung, J. Y., Youngblood, J. P. \& Stafford, C. M. 2007 Anisotropic wetting on tunable microwrinkled surfaces. Soft Matt. 3, 1163-1169. 
Cox, R. G. 1983 The spreading of a liquid on a rough solid surface. J. Fluid Mech. 131, 1-26.

Cramér, H. 1962 Random Variables and Probability Distributions, 2nd edn. Cambridge University Press.

Ehrhard, P. \& Davis, S. H. 1991 Non-isothermal spreading of liquid drops on horizontal plates. J. Fluid Mech. 229, 365-388.

Gardiner, C. W. 1985 Handbook of Stochastic Methods. Springer.

Gaskell, P. H., Jimack, P. K., Sellier, M. \& Thompson, H. M. 2004 Efficient and accurate time adaptive multigrid simulations of droplet spreading. Intl J. Numer. Meth. Fluids 45, 1161-1186.

Gramlich, C. M., Mazouchi, A. \& Homsy, G. M. 2004 Time-dependent free surface Stokes flow with a moving contact line. II. Flow over wedges and trenches. Phys. Fluids 16, 1660-1667.

Hocking, L. M. 1983 The spreading of a thin drop by gravity and capillarity. Q. J. Mech. Appl. Math. 36, 55-69.

Huh, C. \& Scriven, L. E. 1971 Hydrodynamic model of steady movement of a solid/liquid/fluid contact line. J. Colloid Interface Sci. 35 (1), 85-101.

Kalliadasis, S. 2000 Nonlinear instability of a contact line driven by gravity. J. Fluid Mech. 413, 355-378.

Katzav, E., Adda-Bedia, M., Amar, M. Ben \& Boudaoud, A. 2007 Roughness of moving elastic lines: crack and wetting fronts. Phys. Rev. E 76, 051601.

Moulinet, S., Guthmann, C. \& Rolley, E. 2002 Roughness and dynamics of a contact line of a viscous fluid on a disordered substrate. Eur. Phys. J. E 8, 437-443.

NikolayeV, V. S. 2005 Dynamics and depinning of the triple contact line in the presence of periodic surface defects. J. Phys. Condens. Matter 17, 2111-2119.

Rednikov, A. Y., Rossomme, S. \& Colinet, P. 2009 Steady microstructure of a contact line for a liquid on a heated surface overlaid with its pure vapor: parametric study for a classical model. Multiphase Sci. Technol. 21, 213-248.

RicE, S. O. 1939 The distribution of maxima of a random curve. Am. J. Math. 61, 409-416.

RiCE, S. O. 1945 The mathematical analysis of random noise. Bell Syst. Tech. J. 24, 46-156.

SaVVa, N. \& Kalliadasis, S. 2009 Two-dimensional droplet spreading over topographical substrates. Phys. Fluids 21, 092102.

Savva, N., Pavliotis, G. A. \& Kalliadasis, S. 2011 Contact lines over random topographical substrates. Part 1. Statics. J. Fluid Mech. 672, 358-383.

SCHWARTZ, L. W. 1998 Hysteretic effects in droplet motions on heterogeneous substrates: direct numerical simulation. Langmuir 14, 3440-3453.

SChwartz, L. W. \& Eley, R. R. 1998 Simulation of droplet motion on low-energy and heterogeneous surfaces. J. Colloid Interface Sci. 202, 173-188.

SodtKe, C., Ajaev, V. S. \& Stephan, P. 2008 Dynamics of volatile liquid droplets on heated surfaces: theory versus experiment. J. Fluid Mech. 610, 343-362.

Tanguy, A. \& Vettorel, T. 2004 From weak to strong pinning. I. A finite size study. Eur. Phys. J. B 38, 71-82.

Troian, S., Herbolzheimer, S., Safran, S. \& Joanny, J. 1989 Fingering instabilities of driven spreading films. Europhys. Lett. 10, 25-39. 